\title{
The Integrated UPR and ERAD in Oligodendrocytes Maintain Myelin Thickness in Adults by Regulating Myelin Protein Translation
}

\author{
Shuangchan Wu, ${ }^{1,2}$ Sarrabeth Stone, ${ }^{1,2}{ }^{\oplus}$ Klaus-Armin Nave, ${ }^{3}$ and ${ }^{\oplus}$ Wensheng Lin ${ }^{1,2}$ \\ ${ }^{1}$ Department of Neuroscience, ${ }^{2}$ Institute for Translational Neuroscience, University of Minnesota, Minneapolis, Minnesota 55455, and ${ }^{3}$ Department \\ of Neurogenetics, Max Planck Institute of Experimental Medicine, D-37075 Göttingen, Germany
}

Myelin proteins, which are produced in the endoplasmic reticulum (ER), are essential and necessary for maintaining myelin structure. The integrated unfold protein response (UPR) and ER-associated degradation (ERAD) are the primary ER quality control mechanism. The adaptor protein Sel1L (Suppressor/Enhancer of Lin-12-like) controls the stability of the E3 ubiquitin ligase Hrd1 (hydroxymethylglutaryl reductase degradation protein 1), and is necessary for the ERAD activity of the Sel1LHrd1 complex. Herein, we showed that SellL deficiency specifically in oligodendrocytes caused ERAD impairment, the UPR activation, and attenuation of myelin protein biosynthesis; and resulted in late-onset, progressive myelin thinning in the CNS of adult mice (both male and female). The pancreatic ER kinase (PERK) branch of the UPR functions as the master regulator of protein translation in ER-stressed cells. Importantly, PERK inactivation reversed attenuation of myelin protein biosynthesis in oligodendrocytes and restored myelin thickness in the CNS of oligodendrocyte-specific Sel1L-deficient mice (both male and female). Conversely, blockage of proteolipid protein production exacerbated myelin thinning in the CNS of oligodendrocytespecific Sel1L-deficient mice (both male and female). These findings suggest that impaired ERAD in oligodendrocytes reduces myelin thickness in the adult CNS through suppression of myelin protein translation by activating PERK.

Key words: ERAD; myelin thickness; oligodendrocyte; PERK; protein translation; UPR

Significance Statement

Myelin is an enormous extended plasma membrane of oligodendrocytes that wraps and insulates axons. Myelin structure, including thickness, was thought to be extraordinarily stable in adults. Myelin proteins, which are produced in the endoplasmic reticulum (ER), are essential and necessary for maintaining myelin structure. The integrated unfolded protein response (UPR) and ER-associated degradation (ERAD) are the primary mechanism that maintains ER protein homeostasis. Herein, we explored the role of the integrated UPR and ERAD in oligodendrocytes in regulating myelin protein production and maintaining myelin structure using mouse models. The results presented in this study imply that the integrated UPR and ERAD in oligodendrocytes maintain myelin thickness in adults by regulating myelin protein production.

Received Mar. 13, 2020; revised Aug. 9, 2020; accepted Sep. 10, 2020.

Author contributions: S.W., S.S., and W.L. designed research; S.W. and S.S. performed research; S.W., S.S., and W.L. analyzed data; S.W., S.S., and K.-A.N. edited the paper; K.-A.N. contributed unpublished reagents/ analytic tools; W.L. wrote the paper.

This work was supported by National Institutes of Health Grants NS094151 and NS105689 to W.L. We thank Dr. Ling Qi (University of Michigan, Ann Arbor, Michigan) for providing the Sel1L ${ }^{\text {loxP/loxP }}$ mice; Dr. M. A. Aryan Namboodiri (Uniformed Services University of the Health Sciences, Bethesda, Maryland) for providing the antibody against aspartoacylase; Dr. Alexander Gow (Wayne State University, Detroit, Michigan) for providing the AA3 antibody against proteolipid protein; and Dr. Wendy Macklin (University of Colorado School of Medicine, Aurora, Colorado) for sending us the PLP KO mice.

The authors declare no competing financial interests.

Correspondence should be addressed to Wensheng Lin at linw@umn.edu.

https://doi.org/10.1523/JNEUROSCI.0604-20.2020

Copyright $\odot 2020$ the authors

\section{Introduction}

Endoplasmic reticulum (ER)-associated degradation (ERAD) is a process that recognizes unfolded or misfolded proteins in the ER, and retrotranslocates these proteins to the cytosol where they are degraded by the ubiquitin-proteasome system (Nakatsukasa et al., 2014; Ruggiano et al., 2014; Qi et al., 2017). Accumulation of unfolded or misfolded proteins in the ER causes ER stress and activation of its stress sensors: pancreatic ER kinase (PERK), inositol requiring enzyme 1 (IRE1), and activating transcription factor $6 \alpha(\mathrm{ATF} 6 \alpha)$. These ER sensors coordinate an adaptive program known as the unfolded protein response (UPR), which restores ER protein homeostasis by facilitating protein folding, attenuating protein translation, and enhancing ERAD (Marciniak and Ron, 2006; Hetz et al., 2015; Wang and Kaufman, 2016). On the other hand, ERAD regulates the UPR by controlling degradation of the ER stress sensors (Horimoto et al., 2013; Sun et al., 2015; 
Sugimoto et al., 2017). The integrated UPR and ERAD are the primary mechanism that maintains ER protein homeostasis (Nakatsukasa et al., 2014; Qi et al., 2017; Hwang and Qi, 2018).

Myelin, an enormous extended plasma membrane of oligodendrocytes, wraps and insulates axons, which facilitates action potential propagation along axons. Myelin thickness is one of the major determinants of the conduction velocity of myelinated axons (Baumann and Pham-Dinh, 2001; Stadelmann et al., 2019). For many decades, one of the defining features of myelin was thought to be the extraordinary stability of its structure, including thickness (Aggarwal et al., 2011; Stadelmann et al., 2019). Indeed, the generally accepted criterion to distinguish between newly generated myelin and originally existing myelin in CNS demyelinating diseases is that newly generated myelin is thinner than originally existing myelin (Franklin and FfrenchConstant, 2008, 2017; Duncan et al., 2018). Intriguingly, evidence is emerging that the thickness of originally existing myelin can change dynamically in adults (Dutta et al., 2018; Williamson and Lyons, 2018; Phillips et al., 2019). Nevertheless, the intrinsic mechanisms by which oligodendrocytes regulate myelin thickness in adults remain unknown.

Data from vanishing white matter disease, a leukodystrophy caused by mutations in eukaryotic translation initiation factor $2 \mathrm{~B}$ (eIF2B) subunits, demonstrate the essential role of protein translation in oligodendrocytes for maintaining myelin structure in the CNS (Pavitt and Proud, 2009; W. Lin, 2015). Upon ER stress, PERK activation inhibits global protein translation by phosphorylating eIF2 $\alpha$, which suppresses the guanine nucleotide exchange activity of eIF2B by forming a nonproductive phosphorylated-eIF2 $\alpha$ (p-eIF2 $\alpha$ )-eIF2B complex (Marciniak and Ron, 2006; Pavitt and Ron, 2012). It is known that the PERK pathway is a major player in regulating oligodendrocyte viability and function in myelin disorders (W. Lin and Popko, 2009; Volpi et al., 2017; W. Lin and Stone, 2020). Notably, a study showed that attenuation of protein translation induced by artificially strong activation of PERK in oligodendrocytes leads to severe myelin loss in young, developing mice and moderate myelin loss in adult mice (Y. Lin et al., 2014a).

Among over 16 membrane-associated E3 ligase complexes involved in the ERAD, the Suppressor/Enhancer of Lin-12-like (Sel1L)-hydroxymethylglutaryl reductase degradation protein 1 (Hrd1) complex is the best-characterized ERAD machinery. The adaptor protein Sel1L controls the stability of the E3 ubiquitin ligase Hrd1, and is necessary for the ERAD activity of the Sel1LHrd1 complex (Sun et al., 2014; Qi et al., 2017; Wu and Rapoport, 2018). Herein, we showed that Sel1L deficiency specifically in oligodendrocytes impaired ERAD and induced adultonset, progressive myelin thinning in the CNS of mice. The progressive myelin thinning induced by Sel1L deficiency in oligodendrocytes was associated with PERK activation and attenuation of myelin protein biosynthesis, and was abrogated by PERK inactivation in oligodendrocytes. Conversely, blockage of proteolipid protein (PLP) production exacerbated myelin thinning induced by Sel1L deficiency in oligodendrocytes. These findings suggest that the impaired ERAD activity of the Sel1L-Hrd1 complex in oligodendrocytes reduces myelin thickness in the adult CNS through suppression of myelin protein translation by activating the PERK branch of the UPR.

\section{Materials and Methods}

Mice. CNP/Cre mice (Lappe-Siefke et al., 2003), Sel1L $L^{\text {loxp }}$ mice (Sun et al., 2014), PERK ${ }^{\text {loxp }}$ mice (Zhang et al., 2002), and PLP heterozygous $\mathrm{KO}\left(P L P^{+/-}\right)$mice (Klugmann et al., 1997) were on the C57BL/6 background. Sel1L $L^{\operatorname{loxP}}$ mice were crossed with $C N P / C r e$ mice, and the resulting progeny were further crossed with $\operatorname{Sel} L^{\operatorname{lox} P}$ mice to obtain Sel1L loxP/loxP $; C N P / C r e$ mice and control mice, including CNP/Cre mice, Sel1L $L^{\text {loxP/loxP }}$ mice, and wild type mice. Moreover, Sel1L ${ }^{\text {loxP }} ; C N P / C r e$ mice were crossed with PERK ${ }^{\text {loxP }}$ mice to generate Sel1L $L^{\text {loxP }} ; P E R K^{\text {loxP }}$; CNP/Cre mice and Sel1L $L^{\text {loxP }} ;$ PERK $K^{\text {loxP }}$ mice. Sel1L ${ }^{\text {loxP }} ;$ PERK ${ }^{\text {loxP }} ; C N P / C r e$ mice were further crossed with $\operatorname{Sel} 1 L^{\operatorname{lox} P} ; P E R K^{\text {loxP }}$ mice to obtain Sel1L $L^{\text {loxP/loxP }} ;$ PERK $K^{\text {loxP/loxP }} ;$ CNP/Cre mice, Sel1L $L^{\text {loxP/loxP }} ;$ CNP/Cre mice, PERK ${ }^{\text {loxP/loxP }}$; CNP/Cre mice, and control mice, including SellL ${ }^{\text {loxP/loxP }}$ mice, PERK ${ }^{\text {loxP/loxP }}$ mice, and CNP/Cre mice. Additionally, Sel1L $L^{\text {loxP }}$; $C N P / C r e$ mice were crossed with $P L P^{+/-}$mice to generate Sel1L $L^{\text {loxP }}$; CNP/Cre; $P L P^{+/-}$mice and Sel1 $L^{\text {loxP }} ; P L P+/ Y$ mice. Sel1 $L^{\text {loxP }}$; CNP/Cre; $P L P^{+/-}$mice were further crossed with SellL $L^{\text {loxP }}$; $P L P+/ Y$ mice to obtain Sel1 $L^{\text {loxP/loxP }} ; C N P / C r e ; P L P^{-1-}$ or Sell $L^{\text {loxP/loxP }}$; CNP/Cre; PLP-/Y mice, Sel1L loxP/loxP CNP/Cre mice, $P L P^{-/-}$or PLP-/ $Y$ mice, and control mice, including Sel1 $L^{\text {loxP/loxP }}$ mice, $C N P / C r e$ mice, or wild type mice. Genotypes were determined by PCR from DNA extracted from tail tips as described previously (Klugmann et al., 1997; Zhang et al., 2002; Lappe-Siefke et al., 2003; Sun et al., 2014). To assess Cre-mediated recombination of floxed alleles in myelin-producing cells of these mice, genomic DNA was isolated from the indicated tissues and PCR was performed as described previously (Zhang et al., 2002; Sun et al., 2014). We monitored mice daily to detect neurologic phenotypes. All animal procedures were conducted in complete compliance with the National Institutes of Health Guide for the care and use of laboratory animals and were approved by the Institutional Animal Care and Use Committee of the University of Minnesota.

Rotarod test. The rotarod apparatus (Panlab/Harvard Apparatus) with a spindle diameter of $3 \mathrm{~cm}$ was used to test motor coordination of mice. Mice were trained by placing them on the rotor rod at a constant speed $(4 \mathrm{rpm})$ for $180 \mathrm{~s} 1$ day before the test. For the trials, mice were placed on the rotor rod at $4 \mathrm{rpm}$, and then the rotor rod was set to accelerate continuously from 4 to $40 \mathrm{rpm}$ over a period of $300 \mathrm{~s}$ and the latency to fall was recorded (falls were detected with a pressure sensitive lever); $180 \mathrm{~s}$ was the maximum time for the trial, and mice that reached this were recorded as $180 \mathrm{~s}$ and removed from the rotarod apparatus.

Immunohistochemistry (IHC). Anesthetized mice were perfused through the left cardiac ventricle with 4\% PFA in PBS, and the tissues were removed. Half sagittal brains and the cephalic half of the cervical spinal cords were postfixed in $4 \%$ PFA for $2 \mathrm{~h}$, cryoprotected in $30 \%$ sucrose for $48 \mathrm{~h}$, embedded in optimum cutting temperature compound, and frozen on dry ice. Frozen sections were cut using a cryostat at a thickness of $10 \mu \mathrm{m}$. The other half sagittal brains and the caudal half of the cervical spinal cords were postfixed in $4 \%$ PFA for $72 \mathrm{~h}$, dehydrated through graded alcohols, and embedded in paraffin wax. Paraffin sections were cut using a microtome at a thickness of $5 \mu \mathrm{m}$. Fluorescein (1:100, Vector Laboratories, anti-mouse, RRID:AB 11213281, anti-rabbit, RRID:AB_2336197), Cy3 (1:500, Millipore, anti-mouse, RRID:AB_11213281, anti-rat, RRID: AB_90854, antirabbit, RRID:AB_92489), or enzyme-labeled secondary antibodies (1:200, Vector Laboratories, anti-mouse, RRID:AB_2313581) were used for detection. Immunohistochemical detection of CC1 (APC7, 1:50; EMD Biosciences, RRID:AB_2057371), myelin basic protein (MBP, 1:1000; Sternberger Monoclonals, RRID:AB_10120129), aspartoacylase (ASPA, 1:1000, kindly provided by M.A. Aryan Namboodiri at Uniformed Services University of the Health Sciences, Bethesda, MD), Sel1L (1:50, Santa Cruz Biotechnology, catalog \#sc-377351), Hrd1 (1:100, Thermo Fisher Scientific, RRID:AB_2199832), platelet-derived growth factor receptor $\alpha$ (PDGFR $\alpha, 1: 50$, Sigma Millipore, RRID:AB_2283679), proliferating cell nuclear antigen (PCNA,1:200, Santa Cruz Biotechnology, RRID:AB 628110), cleaved caspase 3 (CC3; 1:200, Cell Signaling Technology, RRID: AB_2070042), and CD11b (1:50, Sigma Millipore, RRID:AB_92930) were performed as described previously (Stone et al., 2017, 2018). Fluorescentstained sections were mounted with Vectashield mounting medium with DAPI (Vector Laboratories) and visualized with a Carl Zeiss Axioskop 2 fluorescence microscope or an Olympus FV1000 confocal microscope. Whole-brain sections and spinal cord sections were scanned by a TissueScope LE120 slide scanner (Huron Digital Pathology). Immunopositive cells in the CNS were quantified as 
described by us previously (Y. Lin et al., 2014a,b; Stone et al., 2017, 2018). Only those cells with nuclei observable by DAPI staining were counted.

Electron microscopy (EM) analysis. Mice were anesthetized and perfused with PBS containing 4\% PFA and 2.5\% glutaraldehyde. The cervical spinal cords (C3-C7) were processed and embedded. Ultrathin sections of the spinal cord at C3 level were cut, stained with uranyl acetate and lead citrate, and visualized with a Phillips CM12 transmission EM as described by us previously (Y. Lin et al., 2014b; Stone et al., 2018). We counted the total number of axons, the number of myelinated axons, and measured the diameter of axons and the thickness of myelin in the anterior funiculus medially next to the anterior median fissure in the spinal cord at C3 level using the National Institutes of Health ImageJ software (http://rsb.info.nih.gov/ij/; RRID:SCR_003070) as described by us previously (Y. Lin et al., 2014a; Stone et al., 2018). The g-ratio was calculated as diameter of axon/diameter of axon and myelin.

$B r d U$ pulse-chase analysis. Mice received intraperitoneal injections of $50 \mathrm{mg} / \mathrm{kg} \mathrm{BrdU}$ every other day from the age of 5 to 10 weeks. Mice were anesthetized and perfused with 4\% PFA in PBS. The tissues were removed, postfixed in $4 \% \mathrm{PFA}$, and embedded in optimum cutting temperature compound. Frozen sections were cut using a cryostat at a thickness of $10 \mu \mathrm{m}$. We first performed ASPA or PDGFR $\alpha$ immunostaining as described above. After ASPA or PDGFR $\alpha$ immunostaining, sections were reintroduced to $-20^{\circ} \mathrm{C}$ acetone for $10 \mathrm{~min}$, washed with PBS, incubated with $2 \mathrm{~N} \mathrm{HCl}$ for $60 \mathrm{~min}$ at $37^{\circ} \mathrm{C}$, and neutralized with $0.1 \mathrm{M}$ sodium borate for $10 \mathrm{~min}$ at room temperature. Sections were then incubated with antibody against BrdU (1:250, Sigma Millipore, RRID: AB_476793), followed by secondary antibody (Fluorescein, 1:100, Vector Laboratories, anti-mouse, RRID:AB_11213281) and mounted with Vectashield mounting medium with DAPI (Vector Laboratories). Sections were visualized with a Carl Zeiss Axioskop 2 fluorescence microscope. The number of BrdU-positive cells, ASPA and BrdU double-positive cells, and PDGFR $\alpha$ and BrdU double-positive cells in the cervical spinal cord were quantified as described above.

Western blot analysis. The corpus callosum or half brains (bisected in the sagittal plane) harvested from mice were rinsed in ice-cold PBS and were homogenized using a motorized homogenizer as previously described (W. Lin et al., 2005; Stone et al., 2018). After incubating on ice for $15 \mathrm{~min}$, the extracts were cleared by centrifugation at $14,000 \mathrm{rpm}$ for 30 min twice. The protein content of each extract was determined by DC Protein Assay (Bio-Rad Laboratories). The extracts $(40 \mu \mathrm{g})$ were separated by SDS-PAGE and transferred to nitrocellulose membranes. The membranes were incubated with a primary antibody against Sel1L (1:500, Santa Cruz Biotechnology, catalog \#sc-377351), Hrd1(1:2000, Thermo Fisher Scientific, RRID:AB_2199832), CCAAT/enhancer-binding protein homologous protein (CHOP, 1:1000, Santa Cruz Biotechnology, RRID:AB_ 783507), eukaryotic translation initiation factor $2 \alpha$ (eIF2 $\alpha, 1: 1000$, Santa Cruz Biotechnology, RRID:AB_640075), phosphorylated p-eIF2 $\alpha$ (peIF2 $\alpha, 1: 1000$, Cell Signaling Technology, RRID:AB_330951), ATF6 $\alpha$ (1:500; Cosmo Bio USACA, RRID:AB_10709801), Binding immunoglobulin protein (Bip, 1:500; Santa Cruz Biotechnology, RRID:AB_627698), MBP (1:1000; Sternberger Monoclonals, RRID:AB_10120129), PLP (AA3, 1:100; kindly provided by Alexander Gow, Wayne State University), or $\beta$-actin (1:5000, Sigma Millipore, RRID:AB_476694), followed by an HRP-conjugated secondary antibody (1:1000, Vector Laboratories antimouse, RRID:AB_2336177; anti-rabbit, RIDD:AB_2336198; anti-rat, RRID:AB_2336203). The chemiluminescent signal was detected using the ECL Detection Reagents (GE Healthcare Biosciences). The intensity of the chemiluminescence signals was quantified using the National Institutes of Health ImageJ software. The intensity of individual proteins was normalized to $\beta$-actin. The intensity of $\mathrm{p}$-eIF $2 \alpha$ was normalized to eIF $2 \alpha$ and then to $\beta$-actin.

Measurement of myelin protein biosynthesis with immunoprecipitation-surface sensing of translation (IP-SUnSET). Mice received intraperitoneal injections of puromycin $(0.04 \mu \mathrm{mol} / \mathrm{g}$ body weight) for $6 \mathrm{~h}$ before tissue harvest as described previously (Lee et al., 2017). Proteins were extracted from the half brains (bisected in the sagittal plane) of these mice and quantified by DC Protein Assay (Bio-Rad Laboratories) as described above. Puromycin-labeled, newly synthesized proteins were pulled down by IP using an antibody against puromycin (1:5000, EMD Millipore, RRID:AB_2566826) using the Pierce Direct IP Kit (Thermo Fisher Scientific, catalog \#26148) according to the manufacturer's instructions. We further performed Western blot analysis to determine the levels of newly synthesized MBP and PLP as described above.

Real-time PCR. Half brains (bisected in the sagittal plane) harvested from mice were rinsed in ice-cold PBS and snap frozen with dry ice. RNA was isolated from the brain using Trizol reagent (Invitrogen) and treated with DNaseI (Invitrogen) to eliminate genomic DNA. Reverse transcription was performed using the iScript cDNA Synthesis Kit (BioRad Laboratories). TaqMan real-time PCR for MBP and PLP was performed with LightCycler 480 Probes Master (Roche Diagnostics) on the LightCycler 480 System (Roche Diagnostics) as described by us previously (W. Lin et al., 2005). Real-time PCR for spliced XBP1 mRNA (XBP1s) was performed with LightCycler 480 SYBR Green I Master (Roche Diagnostics) on the LightCycler 480 System (Roche Diagnostics) as described previously (Yoon et al., 2019).

Experimental design and statistical analysis. The sample size for each individual experiment is listed in the corresponding figure legend. Both male and female mice were used in this study. All data are expressed as mean $\pm \mathrm{SD}$. Comparison between two groups was statistically evaluated by $t$ test using GraphPad Prism 6 (GraphPad Software, RRID:SCR_ 002798). Multiple comparisons were statistically evaluated by the oneway ANOVA with a Tukey's post-test or a two-way ANOVA with a Bonferroni post-test using GraphPad Prism 6. $p$ values $<0.05$ were considered significant.

\section{Results}

Sel1L deficiency specifically in oligodendrocytes induces lateonset, progressive myelin thinning in the CNS of adult mice To determine the role of ERAD in oligodendrocytes, we generated a mouse model that allows for deletion of Sel1L specifically in oligodendrocytes in the CNS. Sel1 $L^{\text {loxP/loxP }}$ mice (Sun et al., 2014) were crossed with CNP/Cre mice (Lappe-Siefke et al., 2003 ) to generate Sel1L $L^{\text {loxP/loxP }}$ CNP/Cre mice (Sel1L cKO mice) and control mice (WT mice), including CNP/Cre mice, Sel1L $L^{\text {loxP/loxP }}$ mice, and wild type mice. PCR analysis of genomic DNA confirmed the deletion of exon 6 of the SellL gene selectively in the CNS and PNS of Sel1L cKO mice, but not in other organs of Sel1L cKO mice or in any organs of WT mice (Fig. 1A). Western blot analysis showed that the protein levels of Sel1L $(p=0.0002)$ and Hrd1 $(p=0.0212)$ were significantly decreased in the corpus callosum of Sel1L cKO mice compared with WT mice (Fig. $1 B, C$ ). ASPA (a marker for oligodendrocytes) and Sel1L double immunostaining showed that the immunoreactivity of Sel1L was detectable in oligodendrocytes and other cells in the CNS of WT mice, but became undetectable selectively in oligodendrocytes of Sel1L cKO mice (Fig. 1D). CC1 (another marker for oligodendrocytes) and Hrd1 double immunostaining also showed that the immunoreactivity of Hrd1 was dramatically decreased selectively in oligodendrocytes in the CNS of Sel1L cKO mice compared with WT mice (Fig. 1E). These data suggest that Sel1L deficiency eliminates the ERAD activity of the Sel1L-Hrd 1 complex in oligodendrocytes.

It has been shown that Sel1L deficiency leads to ER stress and activation of the UPR, including the PERK and IRE1 branches, in cells (Sun et al., 2014, 2015). We determined whether elimination of the Sel1L-Hrd1 complex causes activation of the UPR in oligodendrocytes. IRE1 activation upregulates the expression of genes (including Bip) that enhance protein folding and protein degradation by splicing X-Box Binding Protein 1 (XBP1) mRNA. PERK activation inhibits global protein translation but stimulates the expression of numerous stress-responsive genes (including $\mathrm{CHOP}$ ) by phosphorylating eIF $2 \alpha$. Cleavage of 


\section{A} floxed Sel1L $\frac{1}{2} \frac{2}{3} \frac{3}{-} \frac{5}{-} \frac{6}{-} \frac{7}{-} \frac{9}{-}$ WT

B Sel1L KO
floxed Sel1L

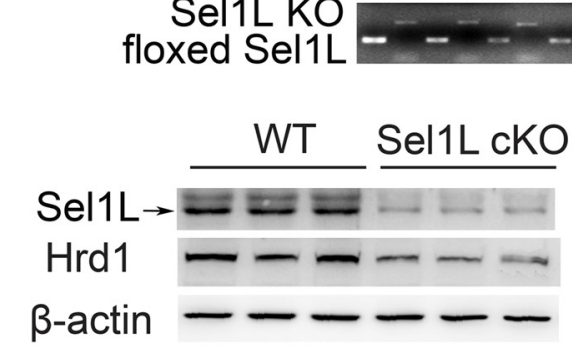

D
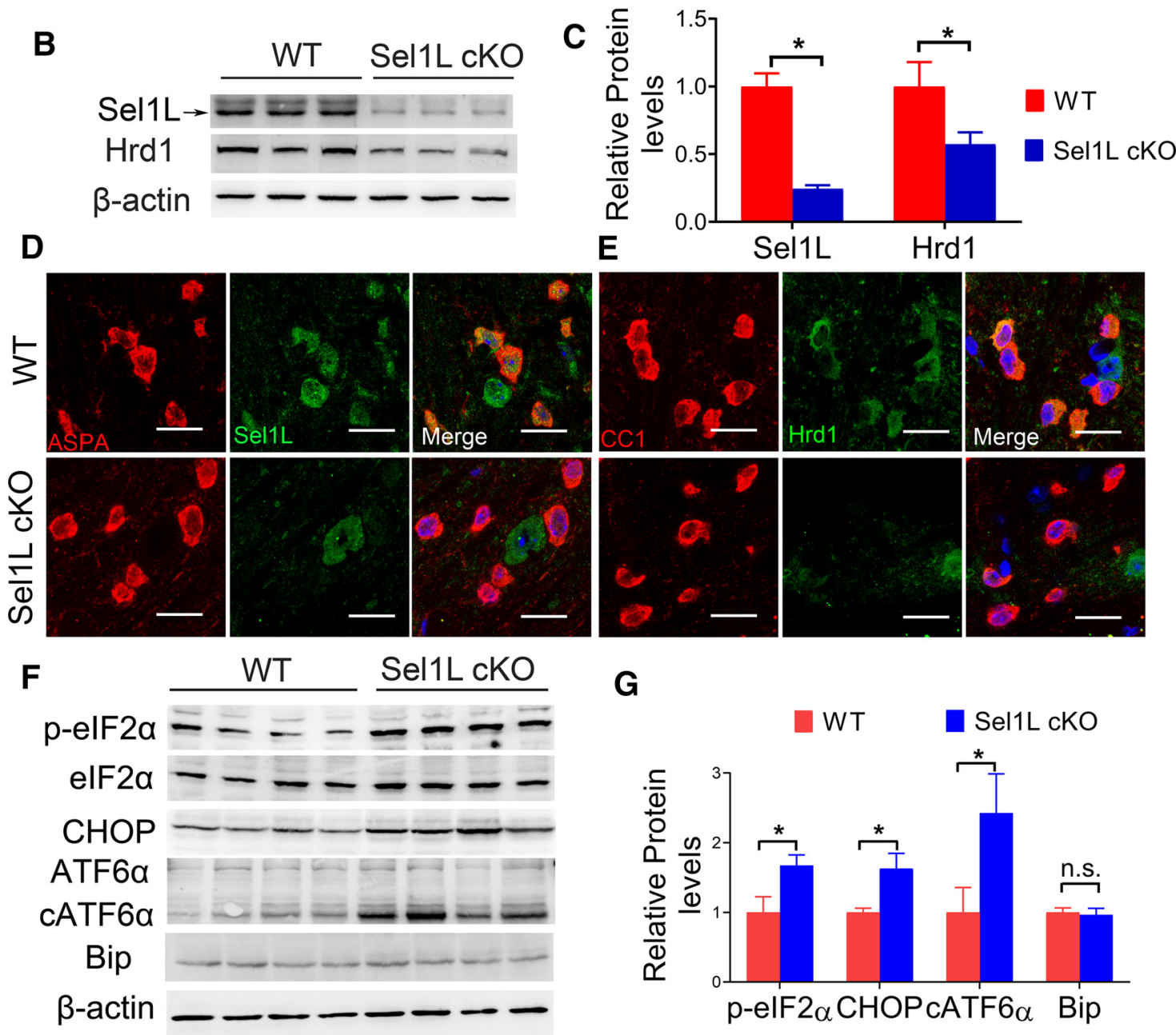
H

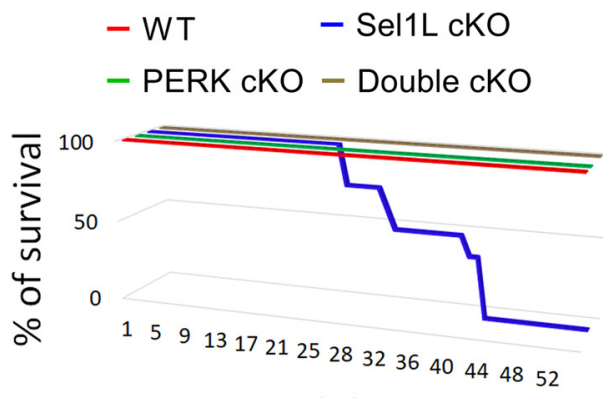

Age $(w)$
G
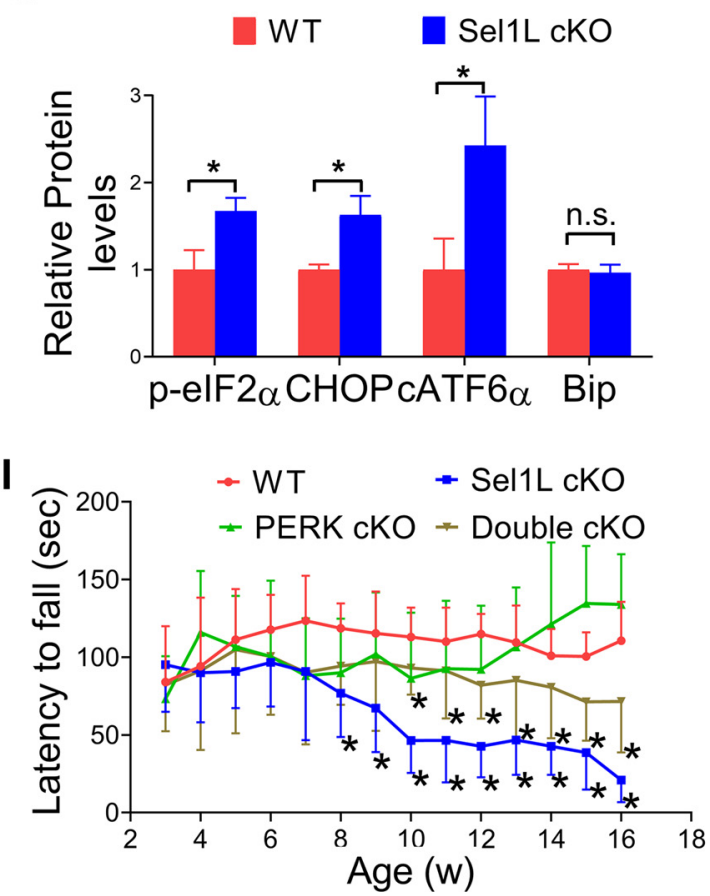

Figure 1. Oligodendrocyte-specific Sel1L-deficient mice developed an adult-onset, progressive tremoring phenotype. $A, P C R$ analysis using genomic DNA showed that the floxed Sel1L allele was present in all tissues in Sel1L cKO mice and WT mice, but the Sel1L KO allele was only present in the CNS and PNS of Sel1L cKO mice. 1, Brain; 2, spinal cord; 3, optic nerve; 4, sciatic nerve; 5, heart; 6, liver; 7, spleen; 8, lung; 9 kidney. $N=3$ animals. $B, C$, Western blot analysis showed that the levels of Sel1L and Hrd1 were significantly decreased in the corpus callosum of 3week(w)-old Sel1L cK0 mice compared with WT mice. $N=3$ animals. $\boldsymbol{D}$, ASPA and Sel1L double immunostaining showed that the immunoreactivity of Sel1L was undetectable selectively in oligodendrocytes of 3-week-old Sel1L cKO mice, compared with WT mice. $N=3$ animals. $E$, $C C 1$ and Hrd1 double immunostaining showed that the immunoreactivity of Hrd1 was dramatically decreased specifically in oligodendrocytes of 3-week-old Sel1L cKO mice compared with WT mice. $N=3$ animals. $\boldsymbol{F}, \mathbf{G}$, Western blot analysis showed that the levels of p-elF $2 \alpha$, CHOP, and cATF6 $\alpha$ were significantly increased in the brain of 3-week-old Sel1L cKO mice compared with WT mice. $N=4$ animals. $\boldsymbol{H}$, Mouse survival curve showed that WT mice, PERK cKO mice, and double cKO mice survived at least 1 year. However, all Sel1L cKO mice died by 43 weeks. $N=6-10$ animals. $I$, Rotarod test showed that the latency to fall of Sel1L cKO mice was significantly shorter than WT mice starting at the age of 8 weeks, and that the latency to fall of double cKO mice was significantly longer than Sel1L cKO starting at the age of 10 weeks (significant effect of group, $F_{(3,48)}=16.71$; significant effect of age, $F_{(6.593,260.7)}=2.540$; and significant group $\times$ age interaction, $\left.F_{(39,519)}=4.434\right) . N=8-10$ animals. Scale bars, $20 \mu \mathrm{m}$. Statistical analyses were done with a $t$ test or a two-way ANOVA with a Bonferroni post-test. Error bars indicate SD. ${ }^{*} p<0.05$. n.s. not significant. 

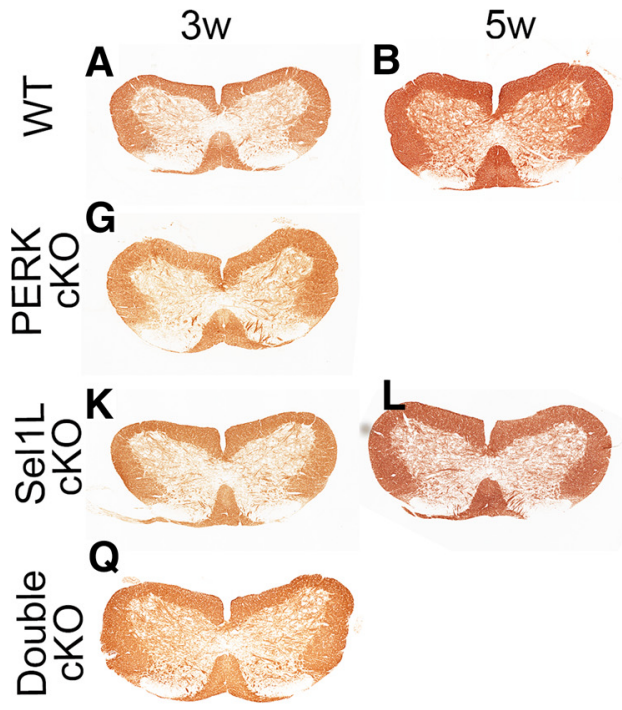
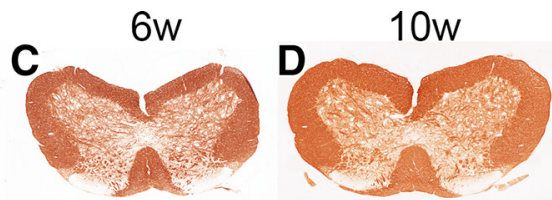

H
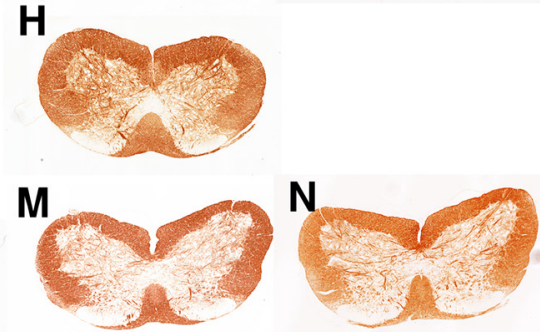

$\mathbf{R}$

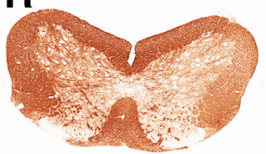

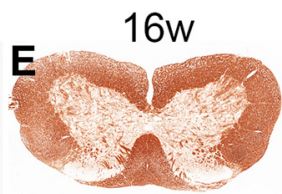
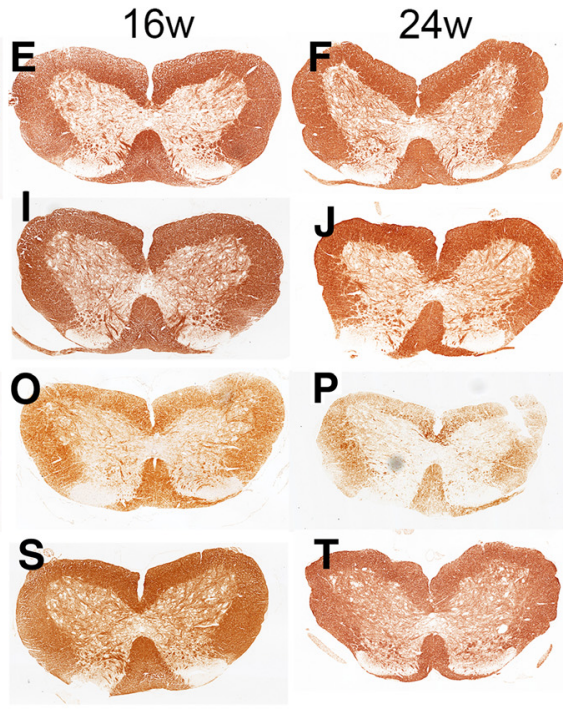

Figure 2. Sel1L cKO mice developed adult-onset myelin loss in the spinal cord, and PERK inactivation in oligodendrocytes rescued myelin loss in the spinal cord of adult Sel1L cKO mice. MBP IHC showed that the immunoreactivity of MBP was not significantly changed in the spinal cord of 3-, 5-, and 6-week-old Sel1L cKO mice compared with WT mice $(\boldsymbol{A}-\boldsymbol{C}, K-M)$, but was noticeably reduced in the spinal cord of 10-, 16-, and 24-week-old Sel1L cKO mice compared with WT mice $(\boldsymbol{D}-\boldsymbol{F}, \mathbf{N}-\boldsymbol{P})$. The immunoreactivity of MBP was comparable in the spinal cord of 3-, 6-, 16-, and 24-week-old PERK CKO mice and WT mice (A-J). Moreover, the immunoreactivity of MBP was not significantly changed in the spinal cord of 3- and 6-week-old double cK0 mice compared with Sel1L cKO mice, PERK CKO mice, and WT mice $(A, C, G, H, K, M, Q, R)$. Importantly, the immunoreactivity of MBP was noticeably increased in the spinal cord of 16- and 24-week-old double cKO mice compared with Sel1L cKO mice $(\boldsymbol{O}, \boldsymbol{P}, \mathbf{S}, \boldsymbol{T})$.

ATF6 $\alpha$ leads to its activation, which enhances the expression of genes that enhance protein folding and protein degradation (Marciniak and Ron, 2006; Hetz et al., 2015; Wang and Kaufman, 2016). Interestingly, Western blot analysis showed that the levels of $\mathrm{p}$-eIF $2 \alpha(p=0.0026), \operatorname{CHOP}(p=0.0015)$, and cleaved ATF6 $\alpha(\operatorname{cATF} 6 \alpha)(p=0.0053)$ were significantly increased in the CNS of Sel1L cKO mice compared with WT mice (Fig. 1F,G). Conversely, real-time PCR analysis showed that the level of spliced XBP1 mRNA (XBP1s) was comparable in the CNS of Sel1L cKO mice and WT mice (see Fig. 8C; $p=0.7857)$. Western blot analysis also showed a comparable level of Bip in the CNS of Sel1L cKO mice and WT mice (Fig. $1 F, G ; p=0.5628)$. These data suggest activation of the PERKeIF $2 \alpha$ branch and the ATF6 $\alpha$ branch, but not the IRE1-XBP1 branch in oligodendrocytes of Sel1L cKO mice. Interestingly, previous studies have shown that ER stress induces activation of PERK and ATF6 $\alpha$, but not IRE1 in oligodendrocytes (Hussien et al., 2015; Stone et al., 2018; W. Lin and Stone, 2020). Thus, these data demonstrate that elimination of the ERAD activity of the Sel1L-Hrd 1 complex disrupts ER protein homeostasis and leads to activation of the UPR in oligodendrocytes.

Surprisingly, young, developing Sel1L cKO mice appeared normal and were indistinguishable from WT mice. Sel1L cKO mice were noticeably smaller than WT mice starting around the age of 6 weeks, and exhibited tremor and ataxia starting around the age of 8 weeks. All Sel1L cKO mice developed severe tremor and died by the age of 43 weeks (Fig. $1 H$ ). Similarly, rotarod test showed that motor coordination in young, developing Sel1L cKO mice appeared normal ( $p=0.1763-0.9933)$, but motor coordination was compromised in adult Sel1L cKO mice starting around the age of 8 weeks ( $p=0.0000-0.0017)$ (Fig. 1I). Therefore, these data demonstrate that Sel1L deficiency specifically in oligodendrocytes causes an adult-onset and progressive tremoring phenotype in mice.

The late-onset tremoring phenotype displayed by Sel1L cKO mice implies myelin abnormalities in the CNS of these mice. MBP IHC showed that there was no obvious decline in MBP immunoreactivity in the CNS white matter of 3-, 5-, and 6-weekold Sel1L cKO mice compared with WT mice (Figs. $2 A-C, K-M$, $3 A-C, K-M)$. Conversely, the immunoreactivity of MBP was noticeably decreased in the CNS white matter of 10-, 16-, and 24-week-old Sel1L cKO mice compared with WT mice (Figs. $2 D-F, N-P, 3 D-F, N-P)$. Moreover, Western blot analysis showed that the levels of MBP ( 3 weeks, $p=0.1732$; 5 weeks, $p=0.8111$ ) and PLP ( 3 weeks, $p=0.7167 ; 5$ weeks, $p=0.1762$ ) were comparable in the CNS of 3- and 5-week-old Sel1L cKO mice and WT mice, but the levels of MBP $(p=0.0015-0.0392)$ and PLP $(p=0.0243-0.0384)$ exhibited a progressive decline in the CNS of Sel1L cKO mice starting at the age of 6 weeks, compared with WT mice (Fig. 3U-W). Accordingly, EM analysis showed that Sel1L deficiency in oligodendrocytes did not alter the gratio ( 3 weeks, $p=0.9999 ; 5$ weeks, $p=0.9999$ ), the thickness of myelin ( 3 weeks, $p=0.9999$; 5 weeks, $p=0.9288$ ), the diameter of axons ( 3 weeks, $p=0.9738$; 5 weeks, $p=0.9726$ ), or the percentage of myelinated axons ( 3 weeks, $p=0.9999 ; 5$ weeks, $p=0.9999)$ in the CNS white matter of 3 - and 5 -week-old mice (Fig. $4 A, B, K, L, U-X$ ). Importantly, EM analysis showed that Sel1 L deficiency in oligodendrocytes led to a progressive increase in the g-ratio ( $p=0.0000-0.0136)$ and a progressive decrease in the myelin thickness $(p=0.0017-0.0399)$ in the CNS white matter starting at the age of 6 weeks, but did not significantly reduce the percentage of myelinated axons until the age of 24 weeks (6-16 weeks, $p=0.8500-0.9999 ; 24$ weeks, $p<0.0001$ ) (Fig. $4 C-F, M-P, U, V, X)$. However, the diameter of axons was comparable in the CNS white matter of Sel1L cKO mice and WT mice at the age of 6, 10, 16, and 24 weeks (Fig. $4 C-F, M-P, W ; p=0.9983-0.9999)$. Collectively, these data suggest that Sel1L deficiency in oligodendrocytes causes progressive thinning of myelin sheaths and eventually complete loss of myelin sheaths in the CNS of adult mice. Since it is known that IHC is not a sensitive assay for quantitative assessment, it is not surprising that modest myelin loss in the CNS of 6week-old Sel1L cKO mice can be detected by Western blot and EM analysis, but not MBP IHC. 

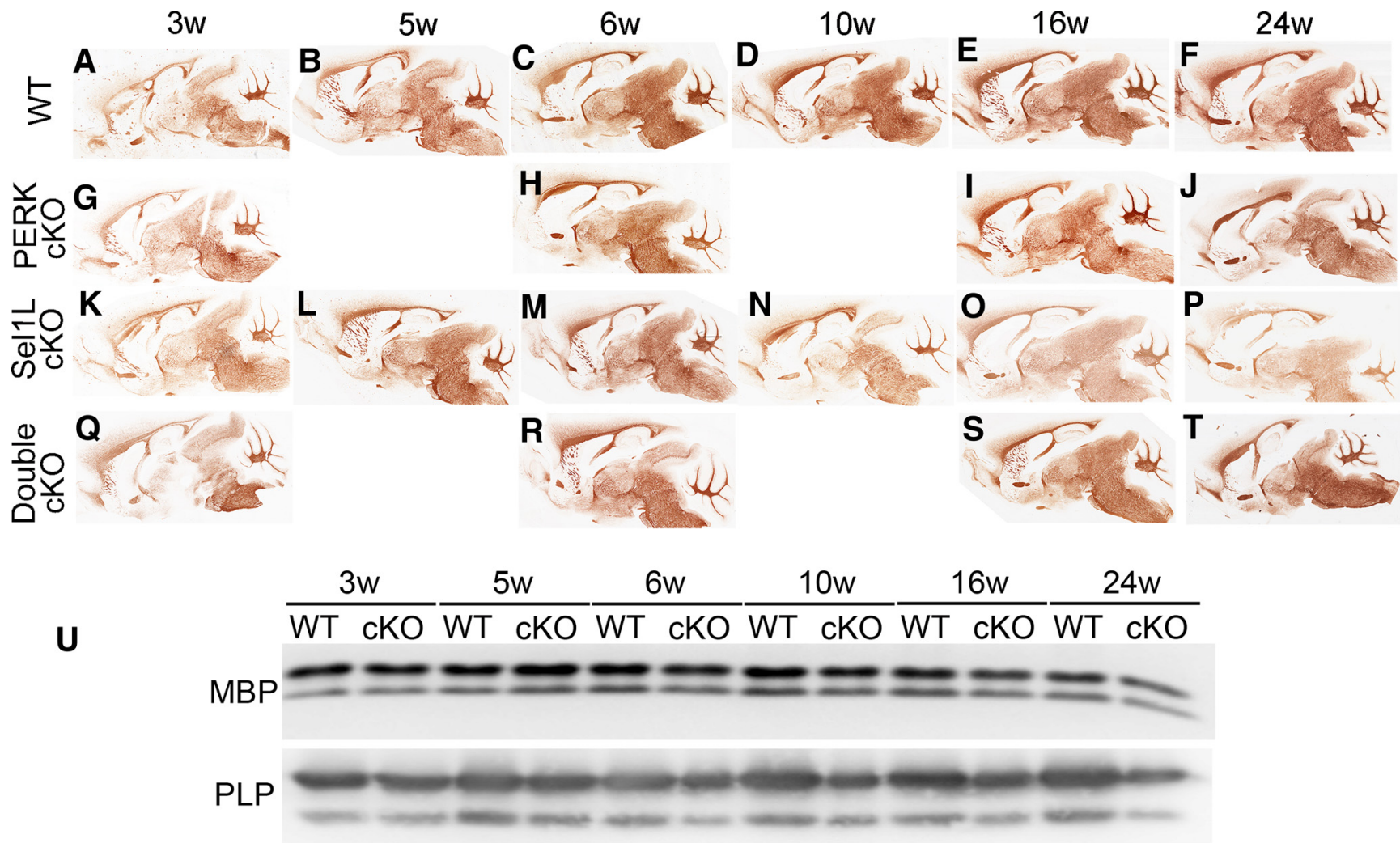

$\beta$-actin
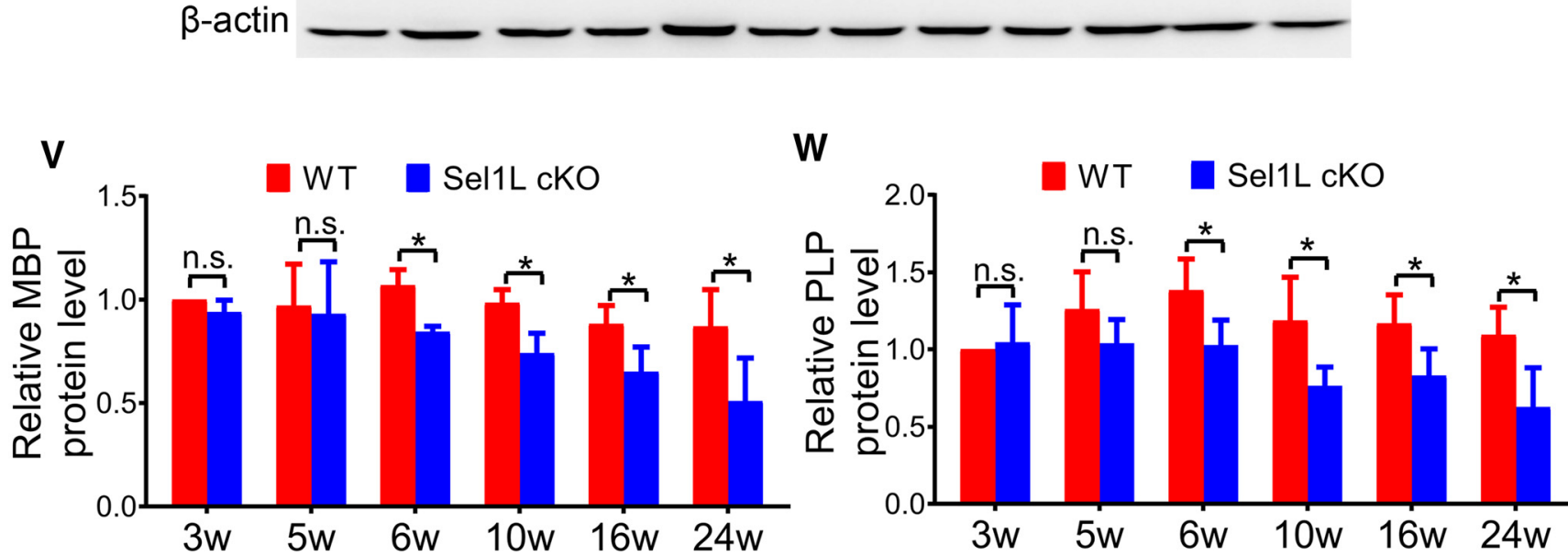

Figure 3. Sel1L cKO mice developed adult-onset myelin loss in the brain, and PERK inactivation in oligodendrocytes rescued myelin loss in the brain of adult Sel1L cKO mice. A-T, MBP IHC showed that the immunoreactivity of MBP was not significantly changed in the brain of 3-, 5-, and 6-week-old Sel1L CKO mice compared with WT mice $(A-C, K-M)$ but was noticeably reduced in the brain of 10-, 16-, and 24-week-old Sel1L cKO mice compared with WT mice (D-F,N-P). The immunoreactivity of MBP was comparable in the brain of 3-, 6-, 16-, and 24-week-old PERK CKO mice and WT mice $(\boldsymbol{A}-\boldsymbol{J})$. Moreover, the immunoreactivity of MBP was not significantly changed in the brain of 3- and 6-week-old double cK0 mice compared with Sel1L cK0 mice, PERK cKO mice, and WT mice $(A, C, G, H, K, M, Q, R)$. Importantly, the immunoreactivity of MBP was noticeably increased in the brain of 16- and 24-week-old double cKO mice compared with Sel1L cKO mice $(\boldsymbol{O}, \boldsymbol{P}, \boldsymbol{S}, \boldsymbol{T})$. $\boldsymbol{U}-\boldsymbol{W}$, Western blot analysis showed that the levels of MBP and PLP were comparable in the brain of 3- and 5-week-old of Sel1L cKO mice and WT mice; however, the levels of MBP and PLP were significantly reduced in the brain of 6-, 10-, 16-, and 24-week-old Sel1L cK0 mice compared with WT mice (MBP: significant effect of group, $F_{(1,6)}=58.4$; significant effect of age, $F_{(5,30)}=5.333$; and no significant group $\times$ age interaction, $F_{(5,30)}=1.544$; PLP: significant effect of group, $F_{(1,6)}=21.86$; significant effect of age, $F_{(2.9,17.4)}=3.334 ;$ and no significant group $\times$ age interaction, $\left.F_{(5,30)}=1.870\right) . N=4$ animals. Statistical analyses were done with a two-way ANOVA with a Bonferroni post-test. Error bars indicate SD. ${ }^{*} p<0.05$. n.s. not significant.

Next, we determined the effects of Sel1L deficiency on oligodendrocyte viability. CC1 and CC3 (a marker for apoptosis) double immunostaining showed that there were a comparable number of oligodendrocytes in the CNS white matter of 3-, 5-, 6-, 10-, and 16-week-old Sel1L cKO mice and WT mice (Fig. $5 A-E, K-O, U-W$; spinal cord, $p=0.1645-0.4224$; corpus callosum, $p=0.1142-0.6777$; cerebellum, $p=0.6140-0.9515$ ). We did not find any CC3-positive oligodendrocytes in the CNS white matter of 3-, 5-, 6-, 10-, and 16-week-old Sel1L cKO mice and WT mice. Interestingly, oligodendrocyte numbers were significantly reduced in the CNS white matter of 24-week-old Sel1L cKO mice compared with WT mice (Fig. 5F,P,U-W; spinal cord, $p=0.0011$; corpus callosum, $p=0.0031$; cerebellum, $p=0.0062$ ). Moreover, we found that CC3-positive oligodendrocytes were undetectable in the CNS white matter of 24-week-old WT mice, but became detectable in Sel1L cKO mice (Fig. 5F,P; 

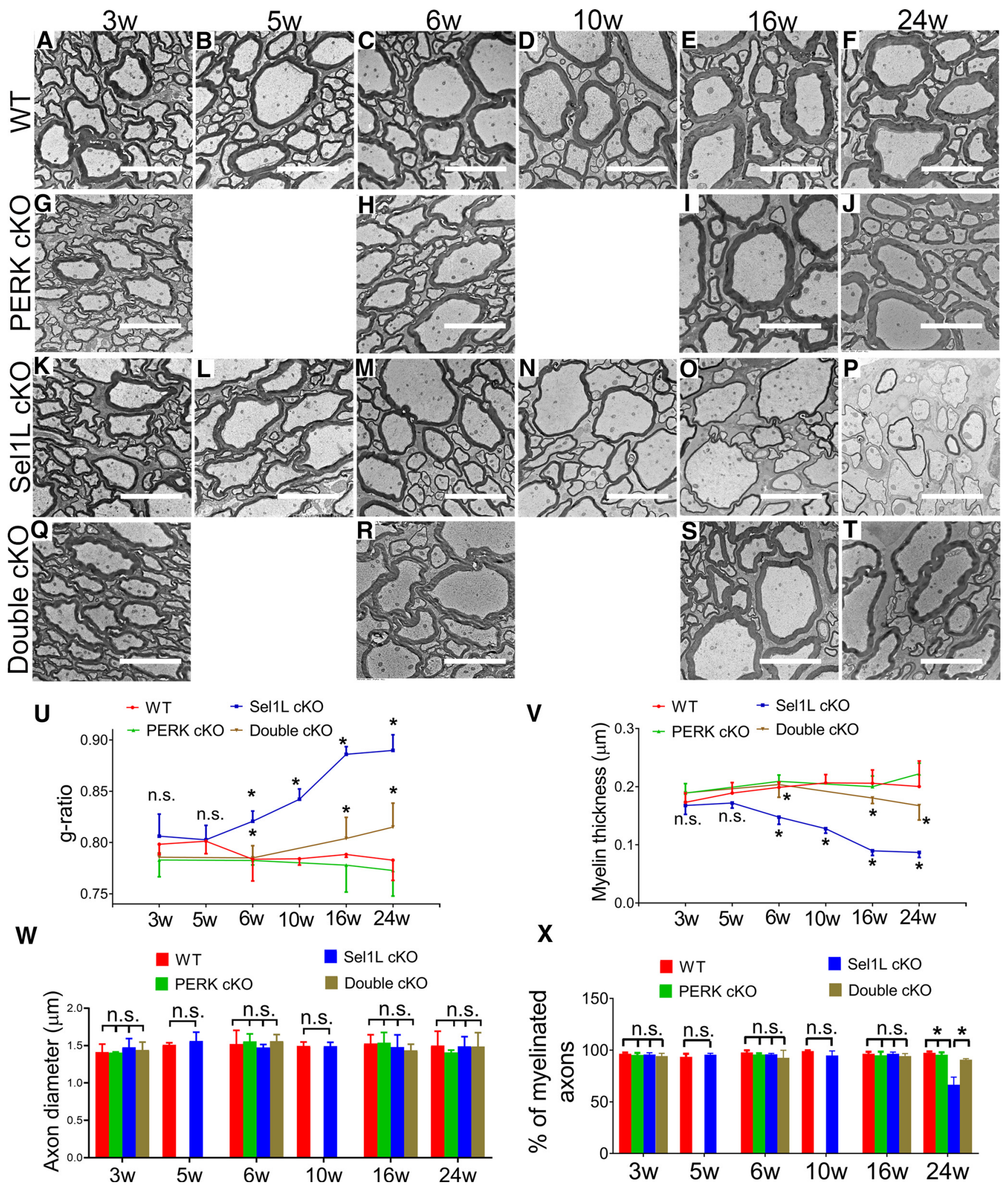

Figure 4. Sel1L cKO mice developed late-onset, progressive myelin thinning in the adult CNS, and PERK inactivation in oligodendrocytes rescued myelin thinning in the CNS of adult Sel1L CKO mice. EM analysis showed that the g-ratio, the thickness of myelin, and the percentage of myelinated axons were comparable in the spinal cord of 3-and 5-week-old Sel1L cK0 mice and WT mice $(A, B, K, L, U, V, X)$; however, a progressive increase of g-ratio $\left(\boldsymbol{C}-\boldsymbol{F}, \boldsymbol{M}-\boldsymbol{P}, \boldsymbol{U}\right.$, significant effect of group, $F_{(1,6)}=116.6$; significant effect of age, $F_{(5,30)}=9.223$; and significant group $\times$ age interaction, $\left.F_{(5,30)}=12.23\right)$ and a progressive decrease of myelin thickness $\left(\boldsymbol{C}-\boldsymbol{F}, \boldsymbol{M}-\boldsymbol{P}, \boldsymbol{V}\right.$, significant effect of group, $F_{(1,6)}=524.4$; significant effect of age, $F_{(2.180,13.08)}=4.602$; and significant group $\times$ age interaction, $\left.F_{(5,30)}=11.85\right)$ were observed in the spinal cord of Sel1L cK0 mice starting at the age of 6 weeks, compared with WT mice. Moreover, the percentage of myelinated axons was significantly decreased in the spinal cord of 24-week-old Sel1L cKO mice compared with WT mice $\left(\boldsymbol{F}, \boldsymbol{P}, \boldsymbol{X}\right.$, significant effect of group, $\boldsymbol{F}_{(1,36)}=53.68$; significant effect of age, $F_{(5,36)}=42.64$; and significant group $\times$ age interaction, $\left.F_{(5,36)}=45.94\right)$. PERK inactivation in oligodendrocytes alone did not alter the g-ratio, myelin thickness, or the percentage of myelinated axons in the spinal cord of 3-, 6-, 16-, and 24-week-old mice $(A-J, U, V, X)$. Importantly, PERK inactivation in oligodendrocytes significantly decreased the g-ratio and increased myelin thickness in the spinal cord of 6-, 16-, and 24-week-old Sel1L cKO mice $(M, 0, P, R-\eta)$, and significantly increased the percentage of myelinated axons in 24-week-old Sel1L cKO mice $(\boldsymbol{P}, T, X)$. Additionally, 

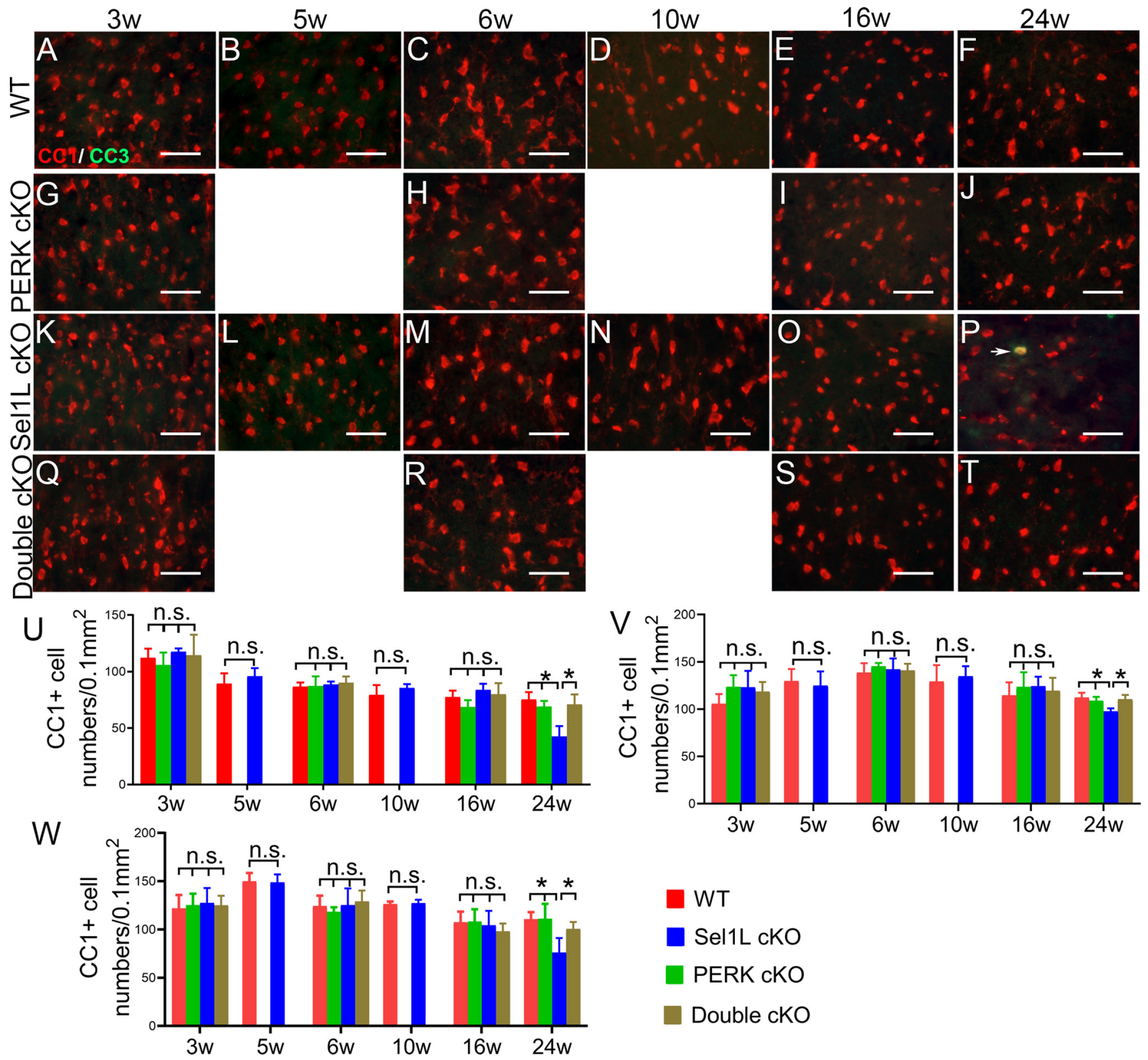

Figure 5. Sel1L cKO mice exhibited oligodendrocyte loss in the CNS at the age of 24 weeks, and PERK inactivation in oligodendrocytes rescued the cell loss in the CNS of Sel1L cKO mice. $\mathrm{CC}_{1}$ and $\mathrm{C}(3$ double immunostaining showed that the number of oligodendrocytes was not significantly changed in the spinal cord $(\boldsymbol{A}-\boldsymbol{E}, \boldsymbol{K}-\boldsymbol{0}, \boldsymbol{U})$, corpus callosum $(\boldsymbol{V})$, and cerebellum $(\boldsymbol{W})$ of 3-, 5-, 6-, 10-, and 16-week-old Sel1L cKO mice compared with WT mice, but was significantly reduced in the spinal cord $(\boldsymbol{F}, \boldsymbol{P}, \boldsymbol{U})$, corpus callosum $(\boldsymbol{V})$, and cerebellum (W) of 24-week-old Sel1L cK0 mice compared with WT mice (spinal cord: 3 weeks, $F_{(3,12)}=0.7436 ; 6$ weeks, $F_{(3,12)}=0.3068 ; 16$ weeks, $F_{(3,12)}=1.95 ; 24$ weeks, $F_{(3,12)}=15.22 ;$ corpus callosum: 3 weeks, $F_{(3,12)}=1.611 ; 6$ weeks, $F_{(3,12)}=0.413 ; 16$ weeks, $F_{(3,12)}=0.430 ; 24$ weeks, $F_{(3,12)}=9.013 ;$ cerebellum: 3 weeks, $F_{(3,12)}=0.123 ; 6$ weeks, $F_{(3,12)}=0.542 ; 16$ weeks, $F_{(3,12)}=0.626 ; 24$ weeks, $\left.F_{(3,12)}=7.67\right)$. PERK inactivation in oligodendrocytes alone did not alter the number of oligodendrocytes in the spinal cord $(\boldsymbol{A}-J, \boldsymbol{U})$, corpus callosum $(\boldsymbol{V})$, and cerebellum $(\boldsymbol{W})$ of 3-, 6-, 16-, and 24-week-old mice. PERK inactivation in oligodendrocytes did not significantly change the number of oligodendrocytes in the spinal cord $(\boldsymbol{K}-\mathbf{0}, \mathbf{Q}-\boldsymbol{S}, \boldsymbol{U})$, corpus callosum $(\boldsymbol{V})$, and cerebellum $(\boldsymbol{W})$ of 3-, 6-, and 16-week-old Sel1L cK0 mice, but significantly increased the number of oligodendrocytes in the spinal cord $(\boldsymbol{P}, \boldsymbol{T}, \boldsymbol{U})$, corpus callosum $(\boldsymbol{V})$, and cerebellum ( $\boldsymbol{W})$ of 24-week-old Sel1L cKO mice. Importantly, there were few CC3-positive oligodendrocytes (arrow) in the spinal cord of 24-week-old Sel1L cK0 mice (P). Scale bars, $50 \mu \mathrm{m}$. $N=4$ animals. Statistical analyses were done with a $t$ test or a one-way ANOVA with a Tukey's post-test. Error bars indicate SD. ${ }^{*} p<0.05$. n.s. not significant.

spinal cord, $0 \pm 0$ vs $1.25 \pm 0.5$ per $0.1 \mathrm{~mm}^{2}, p=0.0011$; corpus callosum, $0 \pm 0$ vs $0.75 \pm 0.5$ per $0.1 \mathrm{~mm}^{2}, p=0.0054$; cerebellum, $0 \pm 0$ vs $0.75 \pm 0.5$ per $0.1 \mathrm{~mm}^{2}, p=0.0054$ ). Conversely, EM analysis showed that both the morphology of oligodendrocytes and

the diameter of axon was comparable in the spinal cord of double cKO mice, Sel1L CKO mice, PERK cK0 mice, and WT mice at the age of 3, 6, 10, 16, and 24 weeks $(A-T, W)$. Scale bars, $5 \mu \mathrm{m}$. $N=4$ animals. Statistical analyses were done with a one-way ANOVA with a Tukey's post-test or a twoway ANOVA with a Bonferroni post-test. Error bars indicate SD. ${ }^{*} p<0.05$. n.s. not significant. the morphology of the ER in these cells in Sel1L cKO mice were indistinguishable from those of control mice at the age of $3,5,6$, 10,16 , and 24 weeks (Fig. $6 A-F, K-P$ ). Together, these results suggest that Sel1L deficiency has a minimal effect on actively myelinating oligodendrocytes during developmental myelination but leads to mature oligodendrocyte dysfunction and eventual death in the CNS of adult mice. It is possible that the minimal effect of Sel1L deficiency on actively myelinating oligodendrocytes results from compensation by other ERAD E3 ligase complexes during developmental myelination. 

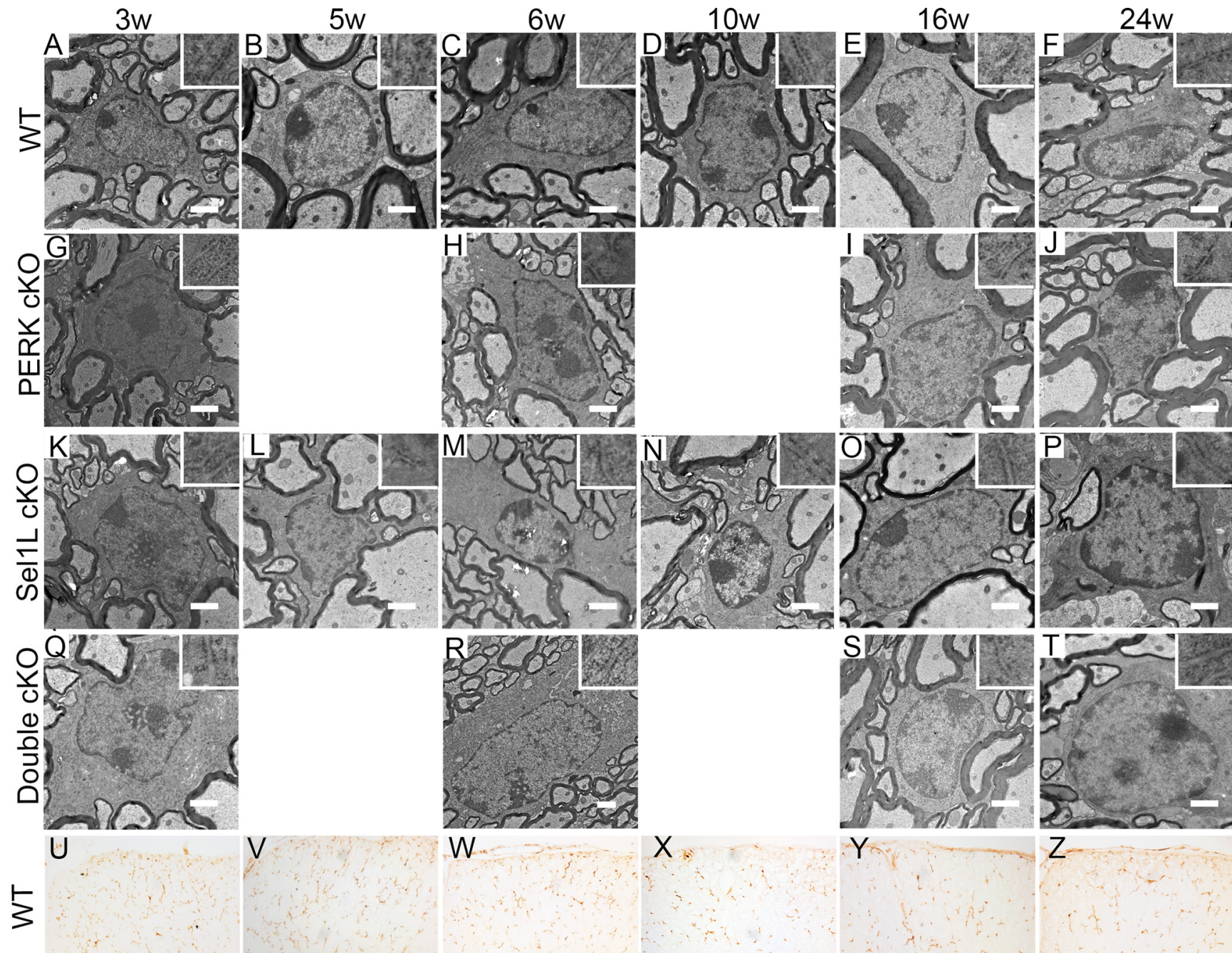

W
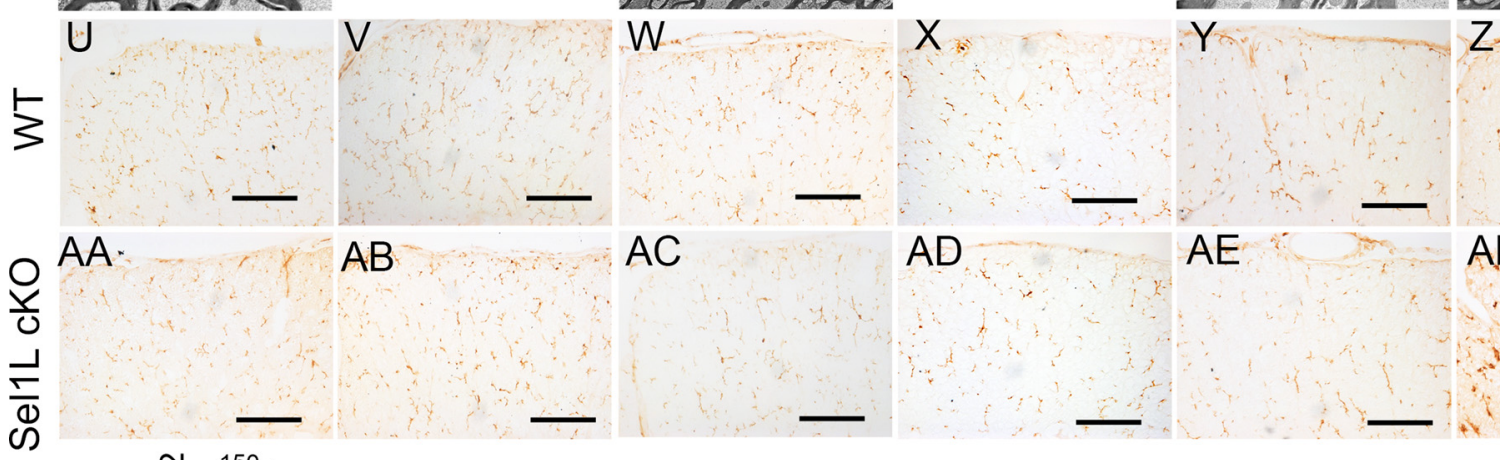

$\mathrm{AC}$
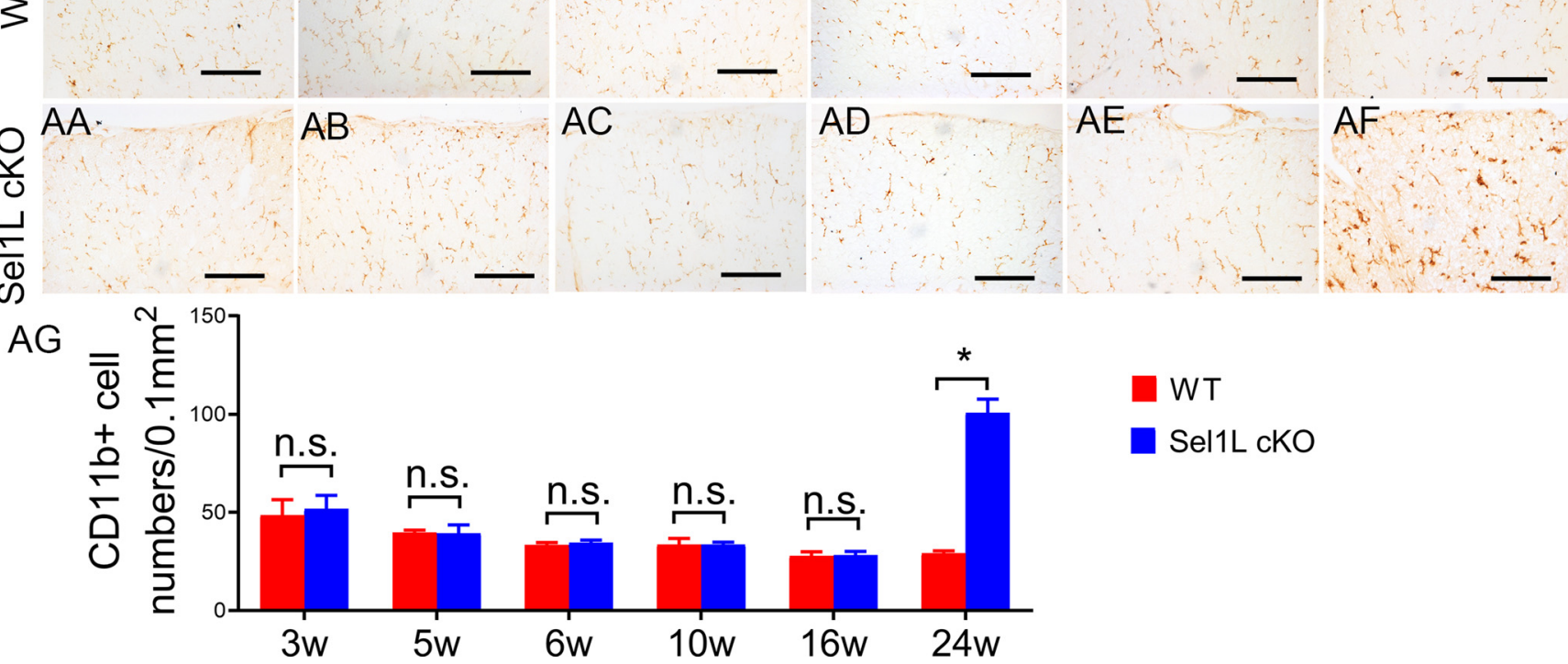

Figure 6. Neither Sel1L deficiency nor PERK inactivation altered the morphology of oligodendrocytes in the CNS. A-T, EM analysis showed that the morphology of oligodendrocytes and the morphology of the ER (insets) in oligodendrocytes appeared normal in the spinal cord of 3-, 5-, 6-, 10-, 16-, and 24-week-old WT mice, PERK cK0 mice, Sel1L cKO mice, and double cKO mice. $U-A G, C D 11 b$ immunostaining showed that the number of microglia was not significantly changed in the spinal cord of 3-, 5-, 6-, 10-, and 16-week-old Sel1L cK0 mice compared with WT mice $(\boldsymbol{U}-Y, A A-A E, A G)$, but was significantly increased the spinal cord of 24-week-old Sel1L cKO mice compared with WT mice $\left(Z, A F, A G\right.$; significant effect of group, $F_{(1,6)}=122.3 ;$ significant effect of age, $F_{(1.877,11.26)}=86.68$; and significant group $\times$ age interaction, $\left.F_{(5,30)}=97.57\right)$. Scale bars: $A-I, 1 \mu \mathrm{m} ; \boldsymbol{U}-\boldsymbol{A F}, 100 \mu \mathrm{m}$. $N=4$ animals. Statistical analyses were done with a twoway ANOVA with a Bonferroni post-test. Error bars indicate SD. ${ }^{*} p<0.05$.

Currently, using thin myelin as a marker of remyelination in CNS demyelinating diseases is a well-accepted practice (Franklin and Ffrench-Constant, 2008, 2017; Duncan et al., 2018). We sought to exclude the possibility that the thin myelin observed in the CNS of Sel1L cKO mice was caused by oligodendrocyte regeneration and remyelination. As described above, there were no signs of myelin breakdown (no loose or uncompact myelin or no increase of naked axon numbers) or oligodendrocyte death 
(no reduction of oligodendrocyte numbers or no increase of apoptotic oligodendrocytes) in the CNS white matter of Sel1L cKO mice compared with WT mice at the age of 3, 5, 6, 10, and 16 weeks (Figs. 4, 5). Conversely, there was an increase of naked axon numbers, a reduction of oligodendrocyte numbers, and an increase of apoptotic oligodendrocytes in the CNS white matter of 24-week-old Sel1L cKO mice compared with WT mice (Figs. 4, 5) Interestingly, CD11b (a maker for microglia) IHC showed that there was no microglia activation in the CNS white matter of Sel1L cKO mice compared with WT mice at the age of $3,5,6,10$, and 16 weeks (Fig. $6 U-Y$, $A A-A E, A G$; 3-16 weeks, $p=0.2623-9468$ ); however, there was noticeable microglia activation in the CNS white matter of 24week-old Sel1L cKO mice compared with WT mice (Fig. $6 Z$, $A F, A G ; p<0.0001)$. It is well documented that microglia are activated in response to demyelination in CNS demyelinating diseases and that reactive microglia activation is required for removal of myelin debris and subsequent regeneration of oligodendrocytes and myelin in these diseases (Franklin and Ffrench-Constant, 2008, 2017). Thus, these results suggest that there is no myelin breakdown (demyelination) in the CNS white matter of Sel1L cKO mice until the age of 24 weeks. Moreover, we performed BrdU pulse-chase analysis, a routine cell proliferation tracing method (Rivers et al., 2008; Psachoulia et al., 2009; Kang et al., 2010; W. Lin et al., 2013), to assess oligodendroglia regeneration in the CNS of Sel1L cKO mice. Both Sel1L cKO mice and WT mice received intraperitoneal injections of $50 \mathrm{mg} / \mathrm{kg}$ BrdU every other day from the age of 5-10 weeks. BrdU and ASPA double immunostaining showed that there were a few ASPA-positive oligodendrocytes that stained positive for BrdU in the CNS white matter of Sel1L cKO mice and WT mice, and that the number of BrdU and ASPA double-positive cells was comparable in these two groups of mice (Fig. $7 A-C ; p=0.5294$ ). Similarly, $\operatorname{BrdU}$ and PDGFR $\alpha$ (a marker for oligodendrocyte precursor cells [OPCs]) double immunostaining showed that there were a number of PDGFR $\alpha$-positive OPCs that stained positive for BrdU in the CNS white matter of Sel1L cKO mice and WT mice, and that the number of BrdU and PDGFR $\alpha$ doublepositive cells was comparable in these two groups of mice (Fig. 7D-F; $p=0.2887$ ). Additionally, PDGFR $\alpha$ and PCNA (a maker for proliferating cells) double immunostaining showed that the number of OPCs was comparable in the CNS white matter of Sel1L cKO mice and WT mice at the age of 3, 5, 6, 10 , and 16 weeks (3-16 weeks, $p=0.5601-0.9001$ ), and that PCNA-positive OPCs were occasionally observed in the CNS white matter of Sel1L cKO mice and WT mice at the age of 3 weeks ( $<1$ PDGFR $\alpha$ and PCNA double-positive cells per 1 $\mathrm{mm}^{2}$ ), but were undetectable in the CNS white matter of these mice at the age of $5,6,10$, and 16 weeks (Fig. $7 G-K, M-$ $Q, S)$. Both OPC numbers $(p=0.0064)$ and PCNA-positive OPC numbers $\left(0 \pm 0\right.$ vs $1.5 \pm 0.57$ per $\left.0.1 \mathrm{~mm}^{2}, p=0.0138\right)$ were significantly increased in the CNS white matter of 24week-old Sel1L cKO mice compared with WT mice (Fig. $7 \mathrm{~L}$, $R, S)$. These results suggest that the rate of oligodendroglia generation or regeneration in the CNS white matter of Sel1L cKO mice is comparable to WT mice at the age of $3,5,6,10$, and 16 weeks, implying that there is no alteration of regeneration of oligodendrocytes and myelin in the CNS of adult Sel1L cKO mice until the age of 24 weeks. Together, these data rule out the contribution of oligodendrocyte regeneration and remyelination to the presence of thin myelin in adult Sel1L cKO mice.
PERK inactivation rescues the detrimental effects of Sel1L deficiency on mature oligodendrocytes in adult mice

Data from our group and other laboratories have demonstrated that the PERK-eIF2 $\alpha$ pathway is a critical player in regulating oligodendrocyte viability and function under normal and disease conditions (W. Lin and Popko, 2009; Volpi et al., 2017; W. Lin and Stone, 2020; Stone et al., 2020). A number of studies showed that PERK deficiency makes both active (re)myelinating and mature oligodendrocytes vulnerable to inflammation in immune-mediated demyelinating diseases (W. Lin et al., 2005, 2006, 2007; Hussien et al., 2014). A very recent study showed that the impaired UPR in oligodendrocytes via deletion of both PERK, and ATF6 $\alpha$ does not affect the viability and function of actively myelinating oligodendrocytes in young, developing mice but leads to mature oligodendrocyte death and demyelination in young adult mice (Stone et al., 2020). Moreover, our previous studies showed that enhanced PERK activation protects both active (re)myelinating and mature oligodendrocytes against inflammation in immune-mediated demyelinating diseases (W. Lin et al., 2013; Y. Lin et al., 2014b). Conversely, we also reported that artificially strong activation of PERK in oligodendrocytes does not affect their viability but leads to severe myelin loss in young, developing mice and moderate myelin loss in adult mice (Y. Lin et al., 2014a). The elevated levels of p-eIF2 $\alpha$ and CHOP in the CNS of Sel1L cKO mice suggest that Sel1L deficiency leads to activation of the PERK-eIF2 $\alpha$ pathway in oligodendrocytes. We determined the involvement of the PERK-eIF $2 \alpha$ pathway in the detrimental effects of Sel1L deficiency on mature oligodendrocytes in adult mice.

Previous studies have demonstrated PERK inactivation specifically in oligodendrocytes in the CNS of PERK ${ }^{\text {loxP/loxP }}$; $C N P / C r e$ mice (PERK cKO mice), which are phenotypically normal and do not display any evidence of myelin or oligodendrocyte abnormalities in the CNS (Hussien et al., 2014; Stone et al., 2020). PERK cKO mice were crossed with Sel1L cKO mice to generate Sel1L $L^{\text {loxP/loxP }} ;$ PERK $K^{\text {loxP/loxP }}$; CNP/Cre mice (double cKO mice). Western blot analysis showed that the levels of p-eIF $2 \alpha$ and CHOP were significantly increased in the CNS of 3- and 6-week-old Sel1L cKO mice compared with PERK cKO mice ( 3 weeks, p-eIF $2 \alpha, p=0.0034$, CHOP, $p=0.0286 ; 6$ weeks, p-eIF $2 \alpha, p<0.0001$, CHOP, $p<0.0001)$ and WT mice ( 3 weeks, p-eIF $2 \alpha, p=0.001$, CHOP, $p=0.0011$; 6 weeks, p-eIF $2 \alpha, p=0.014$, CHOP, $p<0.0001$ ), and were significantly decreased in double cKO mice compared with Sel1L cKO mice ( 3 weeks, p-eIF $2 \alpha, p=0.0309$, CHOP, $p=0.0299 ; 6$ weeks, p-eIF $2 \alpha, p=0.0011$, CHOP, $p=0.0001$ ) (Fig. $8 A, B, D$, $E)$. Conversely, the level of $\operatorname{cATF} 6 \alpha$ was comparable in the CNS of 3- and 6-week-old double cKO mice and Sel1L cKO mice (Fig. $8 A, B, D, E ; 3$ weeks, $p=0.9997 ; 6$ weeks, $p=0.0513$ ). Moreover, real time PCR analysis showed that the level of XBP1s was comparable in the CNS of 3- and 6-week-old WT mice, PERK cKO mice, and Sel1L cKO mice (3 weeks, $p=0.7856-0.9999 ; 6$ weeks, $p=0.6490-0.9918)$, but was significantly increased in the CNS of double cKO mice compared with Sel1L cKO mice, PERK cKO mice, and WT mice (Fig. $8 C, F$; 3 weeks, $p=0.0001-0.0002 ; 6$ weeks, $p=0.0002-0.0006)$. Similarly, Western blot analysis showed that the level of Bip was comparable in the CNS of 3- and 6-week-old WT mice, PERK cKO mice, and Sel1L cKO mice (3 weeks, $p=0.9999 ; 6$ weeks, $p=0.9948-0.9999)$, but was significantly increased in the CNS of double cKO mice compared with Sel1L cKO mice, PERK cKO mice, and WT mice (Fig. 8A,B,D,E; 3 weeks, 

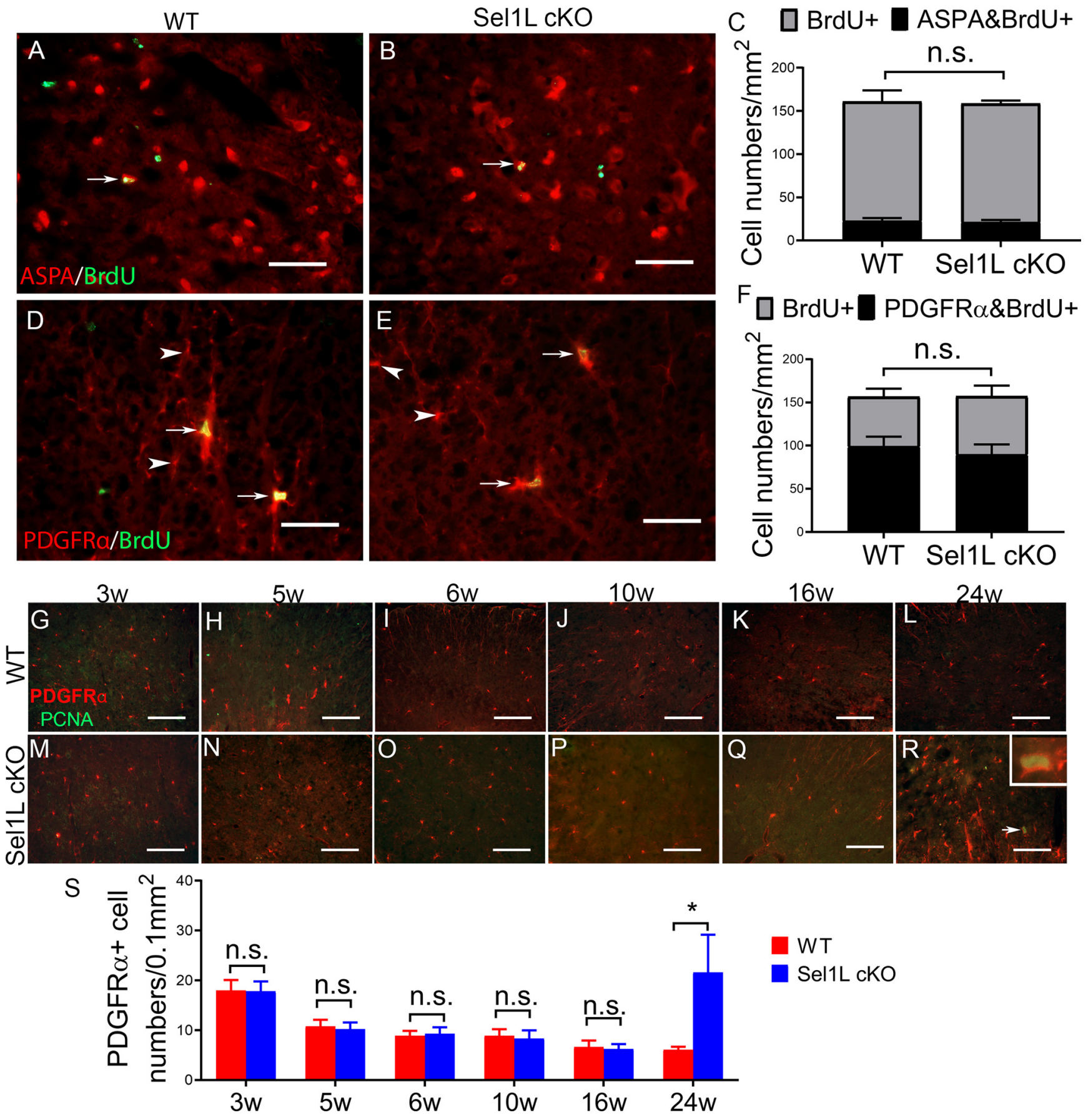

Figure 7. Sel1L deficiency in oligodendrocytes did not alter regeneration of oligodendroglia in the CNS until the age of 24 weeks. A-C, BrdU and ASPA double staining showed a comparable number of BrdU and ASPA double-positive oligodendrocytes (arrow) in the spinal cord of 10-week-old Sel1L cKO mice and WT mice. $\boldsymbol{D}-\boldsymbol{F}$, BrdU and PDGFR $\alpha$ double staining showed a comparable number of BrdU and PDGFR $\alpha$ double-positive OPCs in the spinal cord of 10-week-old Sel1L cKO mice and WT mice. Arrows indicate BrdU and PDGFR $\alpha$ double-positive OPCs. Arrowheads indicate PDGFR $\alpha$-positive OPCs. G-S, PDGFR $\alpha$ and PCNA double immunostaining showed that neither the number of PDGFR $\alpha$ and PCNA double-positive cells nor the number of PDGFR $\alpha$-positive cells was altered in the spinal cord of 3-, 5-, 6-, 10-, and 16-week-old Sel1L cKO mice compared with WT mice. However, both the number of PDGFR $\alpha$ and PCNA double-positive cells (arrow, inset) and the number of PDGFR $\alpha$-positive cells were significantly increased in the spinal cord of 24-week-old Sel1L cKO mice compared with WT mice (significant effect of group, $F_{(1,6)}=11.76$; significant effect of age, $F_{(5,30)}=20.83$; and significant group $\times$ age interaction, $\left.F_{(5,30)}=12.37\right)$. Scale bars: $\boldsymbol{A}, \boldsymbol{B}, \boldsymbol{D}, \boldsymbol{E}, 50 \mu \mathrm{m} ; \boldsymbol{G}-\boldsymbol{R}, 100 \mu \mathrm{m} . N=4$ animals. Statistical analyses were done with a $t$ test or a two-way ANOVA with a Bonferroni post-test. Error bars indicate SD. ${ }^{*} p<0.05$. n.s. not significant.

$p=0.0114-0.0122 ; 6$ weeks, $p<0.0001)$. These results demonstrate abrogation of the PERK-eIF $2 \alpha$ pathway in oligodendrocytes of double $\mathrm{cKO}$ mice.

Similar to Sel1L cKO mice, young, developing double cKO mice did not display any neurologic phenotypes. Intriguingly, adult double cKO mice exhibited noticeably milder tremor and ataxia than Sel1L cKO mice starting around the age of 8 weeks.
Accordingly, rotarod test showed that motor coordination in young, developing double cKO mice appeared normal (double cKO mice vs WT mice, 3-14 weeks, $p=0.6903-0.9997)$. Motor coordination was compromised in adult double $\mathrm{cKO}$ mice starting around age of 15 weeks (double cKO mice vs WT mice, 1516 weeks, $p=0.0392-0.0416$ ); however, the decline in motor coordination in double $\mathrm{cKO}$ mice was significantly milder than 

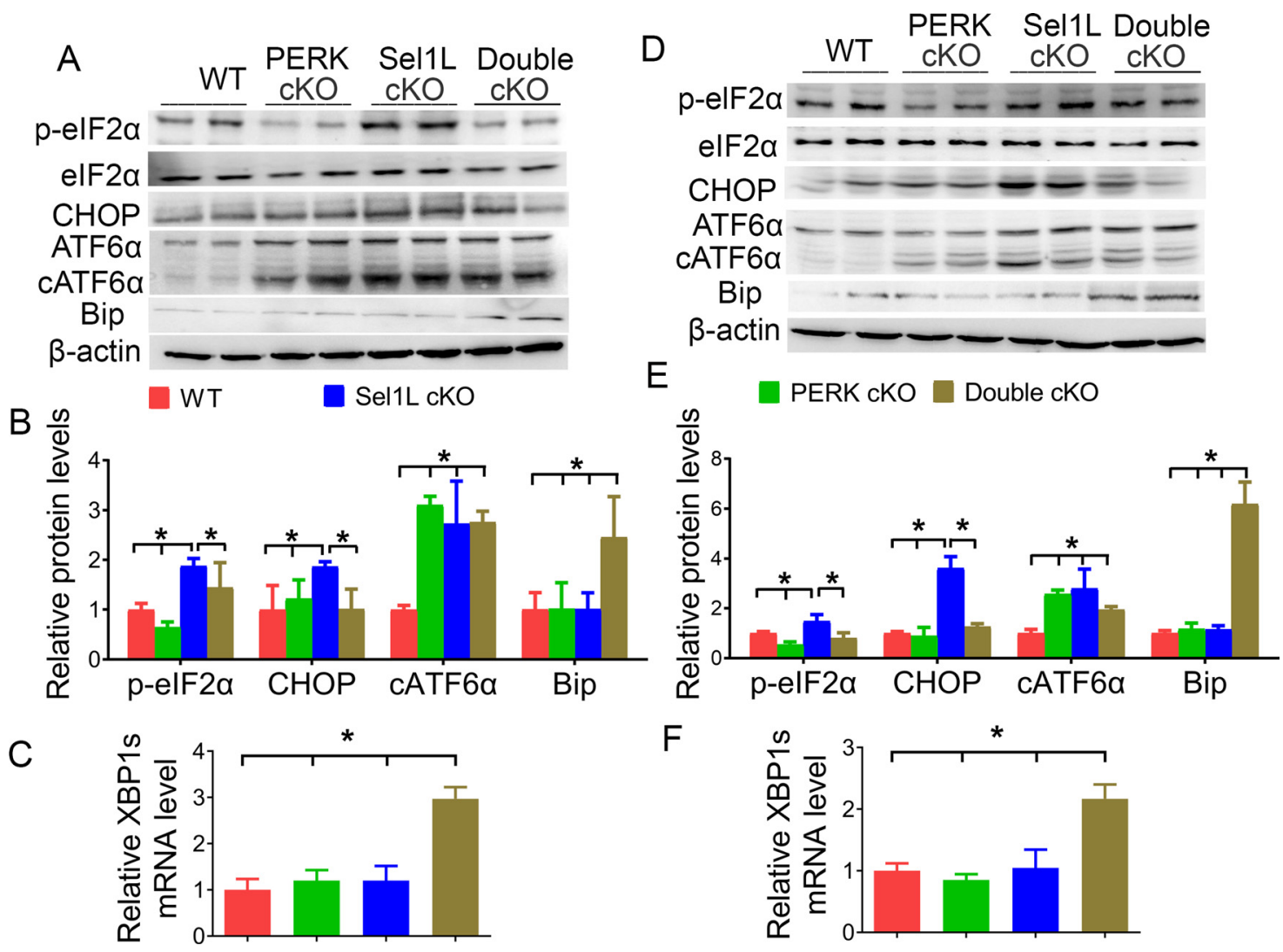

Figure 8. The activity of the PERK-elF2 $\alpha$ pathway was abrogated in oligodendrocytes of double cKO mice. $\boldsymbol{A}, \boldsymbol{B}$, Western blot analysis showed that the levels of $\mathrm{p}$-elF2 $\alpha\left(F_{(3,12)}=20.33\right)$, CHOP $\left(F_{(3,12)}=4.812\right)$, and CATF6 $\alpha\left(F_{(3,12)}=17.42\right)$ were significantly increased in the brain of 3-week-old Sel1L cK0 mice compared with WT mice. Importantly, the levels of p-elF2 $\alpha$ and CHOP were significantly decreased in the brain of 3-week-old double CKO mice compared with Sel1L cKO mice. The level of Bip was significantly increased in the brain of 3-week-old double CKO mice compared with Sel1L CKO mice, PERK CKO mice, and WT mice $\left(F_{(3,12)}=7.188\right)$. C, Real time-PCR analysis showed that the level of XBP1s was significantly increased in the brain of 3-

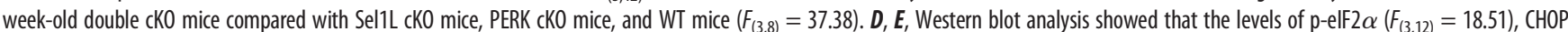
$\left(F_{(3,12)}=75.92\right)$, and CATF6 $\alpha\left(F_{(3,12)}=15.58\right)$ were significantly increased in the brain of 6-week-old Sel1L cKO mice compared with WT mice. Importantly, the levels of p-elF2 $\alpha$ and CHOP were significantly decreased in the brain of 6-week-old double cKO mice compared with Sel1L cKO mice. The level of Bip was significantly increased in the brain of 6-week-old double cKO mice compared with Sel1L cKO mice, PERK $c K 0$ mice, and WT mice $\left(F_{(3,12)}=40.94\right)$. $\boldsymbol{F}$, Real time-PCR analysis showed that the level of XBP1s was significantly increased in the brain of 6 week-old double cKO mice compared with Sel1L cKO mice, PERK cKO mice, and WT mice $\left(F_{(3,8)}=23.88\right)$. $N=4$ animals. Statistical analyses were done with a one-way ANOVA with a Tukey's post-test. Error bars indicate SD. ${ }^{*} p<0.05$.

in age-matched Sel1L cKO mice (double cKO mice vs Sel1L cKO mice, $10-16$ weeks, $p=0.0001-0.0389$ ) (Fig. $1 I$ ). Importantly, in contrast to the premature death of SellL cKO mice, all double cKO mice survived for at least 1 year (Fig. $1 H$ ). Thus, these data demonstrate that PERK inactivation in oligodendrocytes attenuates the tremoring phenotype displayed by Sel1L cKO mice.

The attenuated tremoring phenotype displayed by double cKO mice suggests attenuation of myelin abnormalities in these mice. In accordance with the previous studies (Hussien et al., 2014; Stone et al., 2020), MBP IHC, CC1 and CC3 double immunostaining, and EM analysis showed that oligodendrocytes and myelin appeared normal in the CNS of PERK cKO mice (Figs. 2-6). MBP IHC showed that the immunoreactivity of MBP was comparable in the CNS white matter of 3-and 6-week-old double cKO mice, Sel1L cKO mice, PERK cKO mice, and WT mice (Figs. $2 A, C, G, H, K, M, Q, R, \quad 3 A, C, G, H, K, M, Q, R$ ). Interestingly, the immunoreactivity of MBP was noticeably increased in the CNS white matter of 16- and 24-week-old double cKO mice compared with SellL cKO mice (Figs. 2O,P, $S, T, 3 O, P, S, T)$. EM analysis showed that the percentage of myelinated axons $(p=0.4308)$, the thickness of myelin $(p=$ $0.1657)$, the diameter of axons $(p=0.7173)$, and the g-ratio $(p=0.2121)$ in the CNS white matter of 3-week-old double cKO mice were comparable to SellL cKO mice, PERK cKO mice, and WT mice (Fig. 4A,G,K,Q,U-X). Importantly, EM analysis showed that there was a significant decrease in the gratio (6 weeks, $p=0.0119 ; 16$ weeks, $p=0.0002 ; 24$ weeks, $p=0.0004)$ and a significant increase in myelin thickness $(6$ weeks, $p=0.0007 ; 16$ weeks, $p<0.0001 ; 24$ weeks, $p=0.0335$ ) in the CNS white matter of 6-, 16-, and 24-week-old double cKO mice compared with Sel1L cKO mice, and that the percentage of myelinated axons was significantly increased in 24week-old double cKO mice compared with Sel1L cKO mice $(p=0.0001)$ (Fig. $4 M, O, P, R-V, X)$. However, the diameter of axons was comparable in the CNS white matter of double cKO mice, Sel1L cKO mice, PERK cKO mice, and WT mice at the age of 6,16 , and 24 weeks (Fig. $4 A-T, W$; 6 weeks, $p=0.7203$; 16 weeks, $p=0.6652 ; 24$ weeks, $p=0.8117$ ). These results demonstrate that PERK inactivation in oligodendrocytes rescues progressive myelin thinning in the CNS of adult Sel1L cKO mice. Because of the poor sensitivity of IHC for quantitative assessment, modest differences in myelination in the CNS of 6-week-old Sel1L cKO mice and double cKO mice can be detected by EM analysis, but not by MBP IHC.

We further determined whether PERK inactivation affects oligodendrocyte viability in Sel1 L cKO mice. CC1 and CC3 double immunostaining showed that the number of oligodendrocytes in the CNS white matter of 3-, 6-, and 16-week-old double cKO 
mice was comparable to Sel1L cKO mice, PERK cKO mice, and WT mice (Fig. 5A, $C, E, G-I, K, M, O, Q-S, U-W$; spinal cord, 3 weeks, $p=0.5464 ; 6$ weeks, $p=0.8200 ; 16$ weeks, $p=0.1753$; corpus callosum, 3 weeks, $p=0.2387$; 6 weeks, $p=0.7467$; 16 weeks, $p=0.7352$; cerebellum, 3 weeks, $p=0.9445 ; 6$ weeks, $p=0.6629 ; 16$ weeks, $p=0.6121$ ). Importantly, oligodendrocyte numbers were significantly increased in the CNS white matter of 24-week-old double cKO mice compared with Sel1L cKO mice (Fig. 5P,T-W; spinal cord, $p=0.0010$; corpus callosum, $p=0.0069$; cerebellum, $p=0.0436)$. Moreover, CC3positive oligodendrocytes became undetectable in the CNS white matter of 24-week-old double cKO mice compared with SellL cKO mice (Fig. 5P,T; spinal cord, $0 \pm 0$ vs $1.25 \pm 0.5$ per $0.1 \mathrm{~mm}^{2}$, $p=0.0011$; corpus callosum, $0 \pm 0$ vs $0.75 \pm 0.5$ per $0.1 \mathrm{~mm}^{2}, p=0.0054$; cerebellum, $0 \pm 0$ vs $0.75 \pm 0.5$ per $0.1 \mathrm{~mm}^{2}$, $p=0.0054$ ). Conversely, EM analysis showed that both the morphology of oligodendrocytes and the morphology of the $\mathrm{ER}$ in these cells in double $\mathrm{cKO}$ mice were indistinguishable from those of Sel1L cKO mice, PERK cKO mice, and WT mice at the age of 3,6, 16, and 24 weeks (Fig. 6AT). These results demonstrate that PERK inactivation in oligodendrocytes promotes the cell survival in the CNS of adult Sel1L cKO mice. Together, our data suggest that PERK inactivation rescues mature oligodendrocyte dysfunction and death induced by Sel1L deficiency in adult mice.

\section{Attenuation of myelin protein translation accounts for myelin thinning induced by Sel1L deficiency in oligodendrocytes in adult mice} Our data showed that progressive myelin thinning in adult SellL cKO mice was coupled with the decreased levels of myelin proteins but was not correlated with alterations in oligodendrocyte numbers. Myelin proteins are essential and necessary for maintaining myelin structure (Baumann and Pham-Dinh, 2001; Aggarwal et al., 2011; Stadelmann et al., 2019). The turnover of myelin proteins in the CNS is slow, with the half-life of myelin proteins ranging from days to months (Sabri et al., 1974; Baumann and PhamDinh, 2001). Thus, there is a possibility that slowly progressive myelin thinning in adult Sel1L cKO mice results from the decreased levels of myelin proteins. On the other hand, we showed that PERK inactivation in oligodendrocytes diminished progressive myelin thinning in adult Sel1L cKO mice. The PERK-eIF2 $\alpha$ pathway is the master regulator of protein translation in ER-stressed cells (Marciniak and Ron, 2006; Pavitt and Ron, 2012). Moreover, it is well documented that

G

।

K
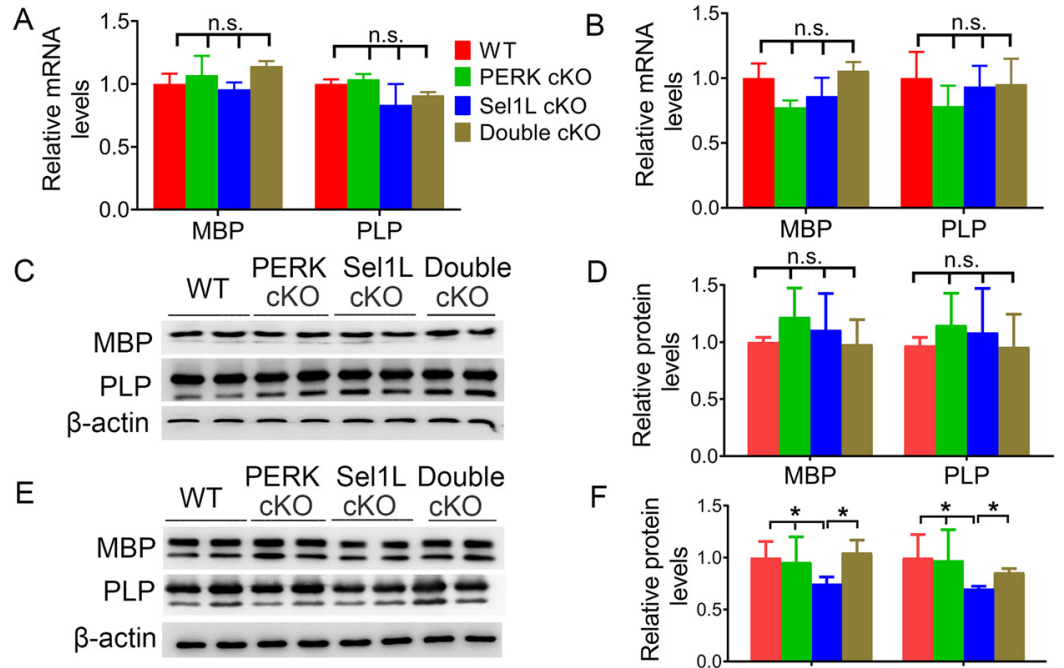

$$
\text { IP: Puromycin }
$$

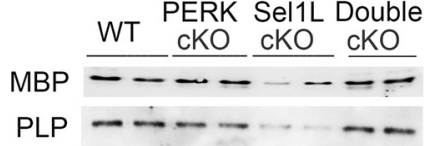

B-actin - $-\ldots-\ldots$
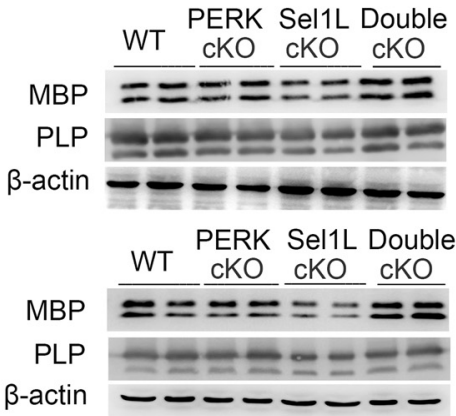

Figure 9. PERK activation in oligodendrocytes suppressed myelin protein biosynthesis in the CNS of Sel1L cKO mice. $A$, Real-time PCR analysis showed that the mRNA levels of $\operatorname{MBP}\left(F_{(3,8)}=2.237\right)$ and $\operatorname{PLP}\left(F_{(3,8)}=3.21\right)$ were comparable in the brain of 3-week-old double cKO mice, Sel1L CKO mice, PERK CKO mice, and WT mice. $\boldsymbol{B}$, Real-time PCR analysis showed that the mRNA levels of $\operatorname{MBP}\left(F_{(3,8)}=3.19\right)$ and $\operatorname{PLP}\left(F_{(3,8)}=1.318\right)$ were comparable in the brain of 6-week-old double cKO mice, Sel1L cKO mice, PERK CKO mice, and WT mice. $C, D$, Western blot analysis showed that the protein levels of MBP $\left(F_{(3,12)}=0.9140\right)$ and PLP $\left(F_{(3,12)}=0.4337\right)$ were comparable in the brain of 3-week-old double cKO mice, Sel1L cKO mice, PERK cKO mice, and WT mice. $\boldsymbol{E}, \boldsymbol{F}$, Western blot analysis showed that the protein levels of $\operatorname{MBP}\left(F_{(3,12)}=8.986\right)$ and PLP $\left(F_{(3,12)}=8.514\right)$ were significantly reduced in the brain of 6-week-old Sel1L cKO mice compared with PERK cKO mice and WT mice. PERK inactivation in oligodendrocytes reversed the reduction of PLP and MBP protein levels in the brain of Sel1L cKO mice. $\boldsymbol{G}, \boldsymbol{H}$, IP-SUnSET analysis showed that the levels of newly synthesized $\operatorname{MBP}\left(F_{(3,12)}=17.43\right)$ and PLP $\left(F_{(3,12)}=\right.$ 10.06) were significantly decreased in the brain of 6-week-old Sel1L cKO mice compared with PERK cKO mice and WT mice. Importantly, PERK inactivation in oligodendrocytes reversed the reduction of newly synthesized PLP and MBP in the brain of Sel1L cK0 mice. I, J, Western blot analysis showed that the protein levels of $\operatorname{MBP}\left(F_{(3,12)}=21.22\right)$ and PLP $\left(F_{(3,12)}=15.78\right)$ were significantly reduced in the brain of 16-week-old Sel1L cKO mice compared with PERK cKO mice and WT mice. PERK inactivation in oligodendrocytes reversed the reduction of MBP and PLP protein levels in the brain of Sel1L cKO mice. $\boldsymbol{K}, \boldsymbol{L}$, Western blot analysis showed that the protein levels of $\operatorname{MBP}\left(F_{(3,12)}=16.10\right)$ and $\operatorname{PLP}\left(F_{(3,12)}=7.061\right)$ were significantly reduced in the brain of 24-week-old Sel1L cKO mice compared with PERK cKO mice and WT mice. PERK inactivation in oligodendrocytes reversed the reduction of MBP and PLP protein levels in the brain of Sel1L cKO mice. $N=4$ animals. Statistical analyses were done with a one-way ANOVA with a Tukey's post-test. Error bars indicate SD. ${ }^{*} p<0.05$. n.s. not significant. impaired protein translation in oligodendrocytes can lead to myelin loss in the CNS (Pavitt and Proud, 2009; W. Lin, 2015). Interestingly, real-time PCR analysis showed that the mRNA levels of MBP (3 weeks, $p=0.1613 ; 6$ weeks, $p=0.0842$ ) and PLP ( 3 weeks, $p=0.0830 ; 6$ weeks, $p=0.3342$ ) were comparable in the CNS of double cKO mice, Sel1L cKO mice, PERK cKO mice, and WT mice at the age of 3 and 6 weeks (Fig. 9A,B). Although 
Western blot analysis showed that the protein levels of MBP $(p=0.4633)$ and PLP $(p=0.7328)$ were comparable in the CNS of double cKO mice, SellL cKO mice, PERK cKO mice, and WT mice at the age of 3 weeks (Fig. 9C,D), the protein levels of MBP and PLP were significantly reduced in the CNS of Sel1L cKO mice compared with PERK cKO mice (MBP, $p=0.0016$; PLP, $p=0.0033$ ) and WT mice (MBP, $p=0.0138$; PLP, $p=0.0079$ ) at the age of 6 weeks (the initiation time point of progressive myelin thinning in Sel1L cKO mice) (Fig. 9E,F). Importantly, PERK inactivation reversed the reduction in protein levels of MBP $(p=0.0016)$ and PLP $(p=0.0164)$ in the CNS of 6-week-old Sel1L cKO mice (Fig. 9E,F). Collectively, these data raise the possibility that attenuation of myelin protein translation induced by PERK activation in mature oligodendrocytes accounts for the reduction of myelin proteins and subsequent myelin thinning in adult Sel1L cKO mice.

To assess the rate of myelin protein translation in oligodendrocytes of adult mice, we used the SUnSET assay, which measure rates of protein biosynthesis by tracking incorporation of puromycin into newly synthesized proteins (Schmidt et al., 2009; Goodman et al., 2011; Lee et al., 2017), in combination with IP. Mice received intraperitoneal injections of puromycin for $6 \mathrm{~h}$ before tissue harvest. Proteins were extracted from the brain of these mice. Puromycin-labeled, newly synthesized proteins were pulled down by IP using the antipuromycin antibody, and then newly synthesized myelin proteins were detected by Western blot. Interestingly, we found that the levels of newly synthesized MBP and PLP were significantly decreased in the CNS of 6-week-old Sel1L cKO mice compared with WT mice (MBP, $p=0.0045$; PLP, $p=0.0079$ ) and PERK cKO mice (MBP, $p=0.0231$; PLP, $p=0.0033$ ) (Fig. 9G,H). Importantly, PERK inactivation in oligodendrocytes abrogated the reduction in newly synthesized MBP $(p=0.0001)$ and PLP $(p=0.0014)$ in the CNS of Sel1L cKO mice (Fig. 9G,H). As mentioned above, both oligodendrocyte numbers and the mRNA levels of MBP and PLP were comparable in the CNS of 6-week-old double cKO mice, SellL cKO mice, PERK cKO mice, and WT mice. Thus, these results demonstrate that PERK activation induced by Sell L deficiency results in attenuation of myelin protein translation in mature oligodendrocytes of adult mice. Moreover, Western blot analysis showed that PERK inactivation in oligodendrocytes reversed the reduction in MBP (16 weeks, $p=0.0059 ; 24$ weeks, $p=0.0006$ ) and PLP (16 weeks, $p=0.0036$; 24 weeks, $p=0.0332$ ) protein levels in the CNS of Sel1L cKO mice at the age of 16 and 24 weeks (Fig. 9I-L). The decreased levels of myelin proteins were correlated with the decreased thickness of myelin in Sel1L cKO mice, and the restored levels of myelin proteins were correlated with the restored thickness of myelin in double cKO mice. Together, our data suggest that PERK activation induced by Sel1L deficiency suppresses myelin protein translation in mature oligodendrocytes, leads to a reduction in myelin protein levels, and subsequently results in progressive myelin thinning in adult mice.

\section{PLP deficiency exacerbates myelin thinning in adult Sel1L cKO mice}

PLP is the most abundant myelin protein in the CNS, which is synthesized in the ER and then transported to myelin sheaths (Baumann and Pham-Dinh, 2001). Evidence suggests that ERAD contributes to degradation of PLP (Karim et al., 2010; Volpi et al., 2017). Data also indicate that intracellular accumulation of PLP can lead to late-onset mature oligodendrocyte death and demyelination in adult mice (Griffiths et al., 1995; Woodward, 2008; Karim et al., 2010). Young adult PLP KO mice (PLP
KO mice) appear healthy and display very subtle myelin abnormalities in the CNS, although aged PLP KO mice show signs of spasticity and develop axon degeneration in the CNS (Klugmann et al., 1997; Griffiths et al., 1998; Rosenbluth et al., 2006). PLP KO mice have been used to study the functions of myelin proteins in oligodendrocyte development, myelin formation, and myelin maintenance, alone or by crossing with mice with deficiency of other main myelin proteins (Klugmann et al., 1997; Uschkureit et al., 2000; Chow et al., 2005). Interestingly, using PLP KO mice, our recent study showed that PLP deficiency rescues late-onset mature oligodendrocyte death and demyelination in mice with double deletion of PERK and ATF6 $\alpha$ in oligodendrocytes (Stone et al., 2020). Thus, we exploited PLP KO mice to determine whether the decreased levels of myelin proteins contribute to myelin thinning in young adult Sel1L cKO mice, and to exclude the possibility that the impaired ERAD activity of the Sel1L-Hrd1 complex in oligodendrocytes leads to intracellular PLP accumulation and subsequently results in late-onset mature oligodendrocyte death and myelin loss in adult Sel1L cKO mice. PLP KO mice were crossed with Sel1L cKO mice to generate Sel1L loxP/loxP $; C N P / C r e ; P L P^{-/-}$mice or Sel1L $L^{\text {loxP/loxP }} ; C N P / C r e$; $P L P-/ Y$ mice (SP DKO mice). Although young, developing SP DKO mice did not display any neurologic phenotypes, adult SPD KO mice exhibited tremor and ataxia starting around the age of 8 weeks. Importantly, the tremoring phenotype deteriorated rapidly, and all SP DKO mice died by 16 weeks (Fig. 10A), compared with Sel1L cKO mice (died by 43 weeks). Moreover, rotarod test showed that motor coordination in young, developing SP DKO mice appeared normal (SP DKO mice vs WT mice, 3-7 weeks, $p=0.3671-0.9968)$. Motor coordination was compromised in adult SP DKO mice starting around the age of 8 weeks (SP DKO mice vs WT mice, 8-14 weeks, $p=0.0003-0.0434$ ). Importantly, the decline in motor coordination in adult SP DKO mice was significantly more severe than in age-matched Sel1L cKO mice (SP DKO mice vs Sel1L cKO mice, 11-14 weeks, $p=$ 0.0004-0.0325) (Fig. 10B). Thus, these data demonstrate that PLP deficiency exacerbates the tremoring phenotype displayed by Sel1L cKO mice and shortens their lifespan.

The exacerbated tremoring phenotype displayed by SP DKO mice suggests exacerbation of myelin loss in these mice. In agreement with previous studies (Klugmann et al., 1997), MBP IHC, CC1 immunostaining, and EM analysis showed that both myelin and oligodendrocytes appeared largely normal in the CNS of 10week-old PLP KO mice (Figs. 10, 11). Interestingly, the immunoreactivity of MBP was noticeably decreased in the CNS white matter of 10-week-old SP DKO mice compared with Sel1L cKO mice (Fig. 10E,F,I,J). Western blot analysis showed that PLP was undetectable in the CNS of 10-week-old PLP KO mice and SP $\mathrm{DKO}$ mice, and that the level of $\operatorname{MBP}(p=0.0075)$ was significantly decreased in the CNS of 10-week-old SP DKO mice compared with SellL cKO mice (Fig. 10K,L). Importantly, EM analysis showed that there was a significant increase in the g-ratio $(p=0.0499)$, a significant decrease in the thickness of myelin $(p<0.0001)$, and a significant decrease in the percentage of myelinated axons $(p<0.0001)$ in the CNS white matter of 10 -weekold SP DKO mice compared with Sel1L cKO mice (Fig. 10M-R, $T)$. Conversely, the diameter of axons was comparable in the CNS white matter of 10-week-old SP DKO mice, Sel1L cKO mice, PLP KO mice, and WT mice (Fig. $10 M-P, S ; p=0.2503$ ). Interestingly, $\mathrm{CC} 1$ immunostaining showed that the number of oligodendrocytes in the CNS white matter of 10-week-old SP DKO mice was comparable to Sel1L cKO mice, PLP KO mice, and WT mice (Fig. 11A-E; spinal cord, $p=0.3389$; corpus 

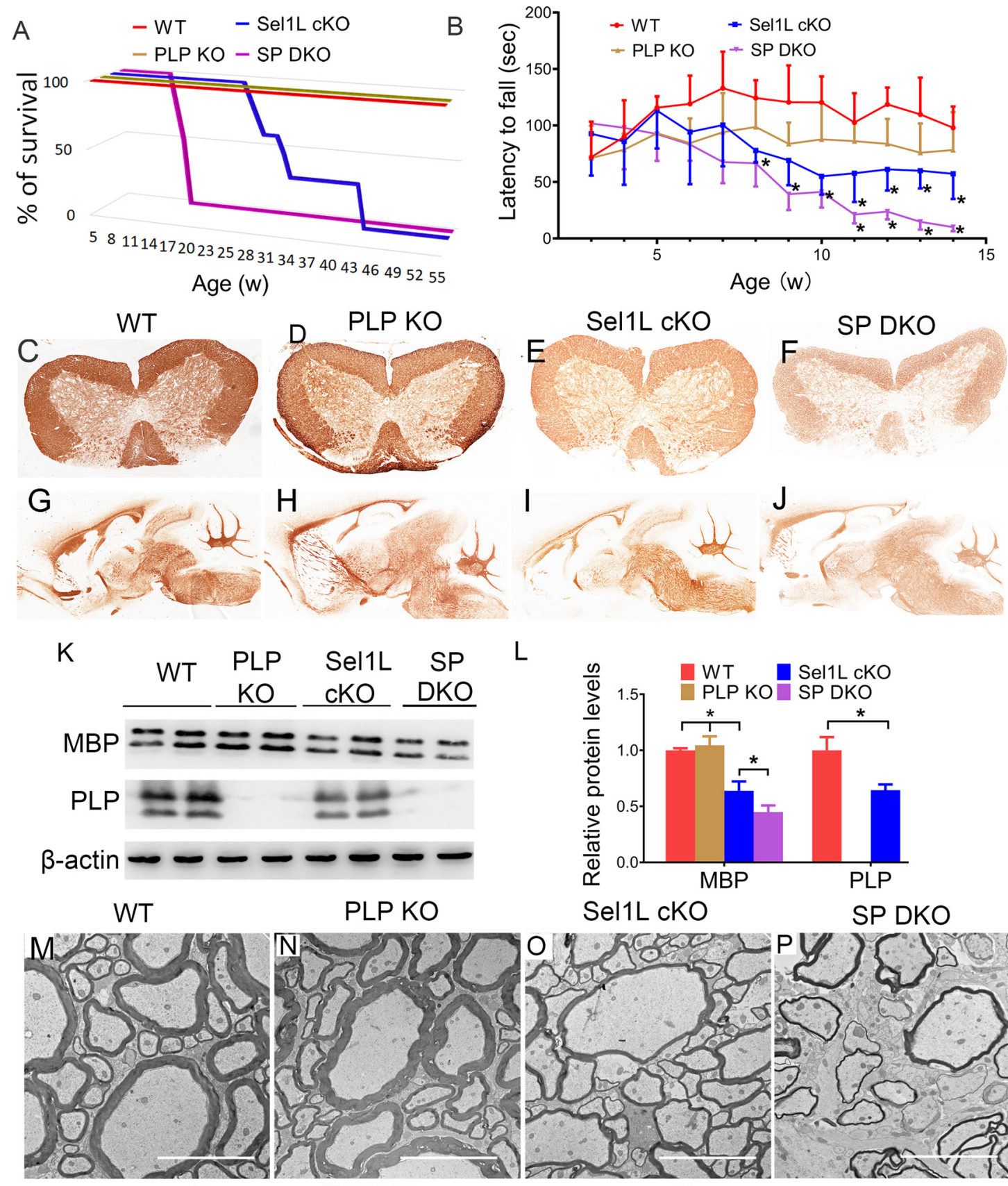

L
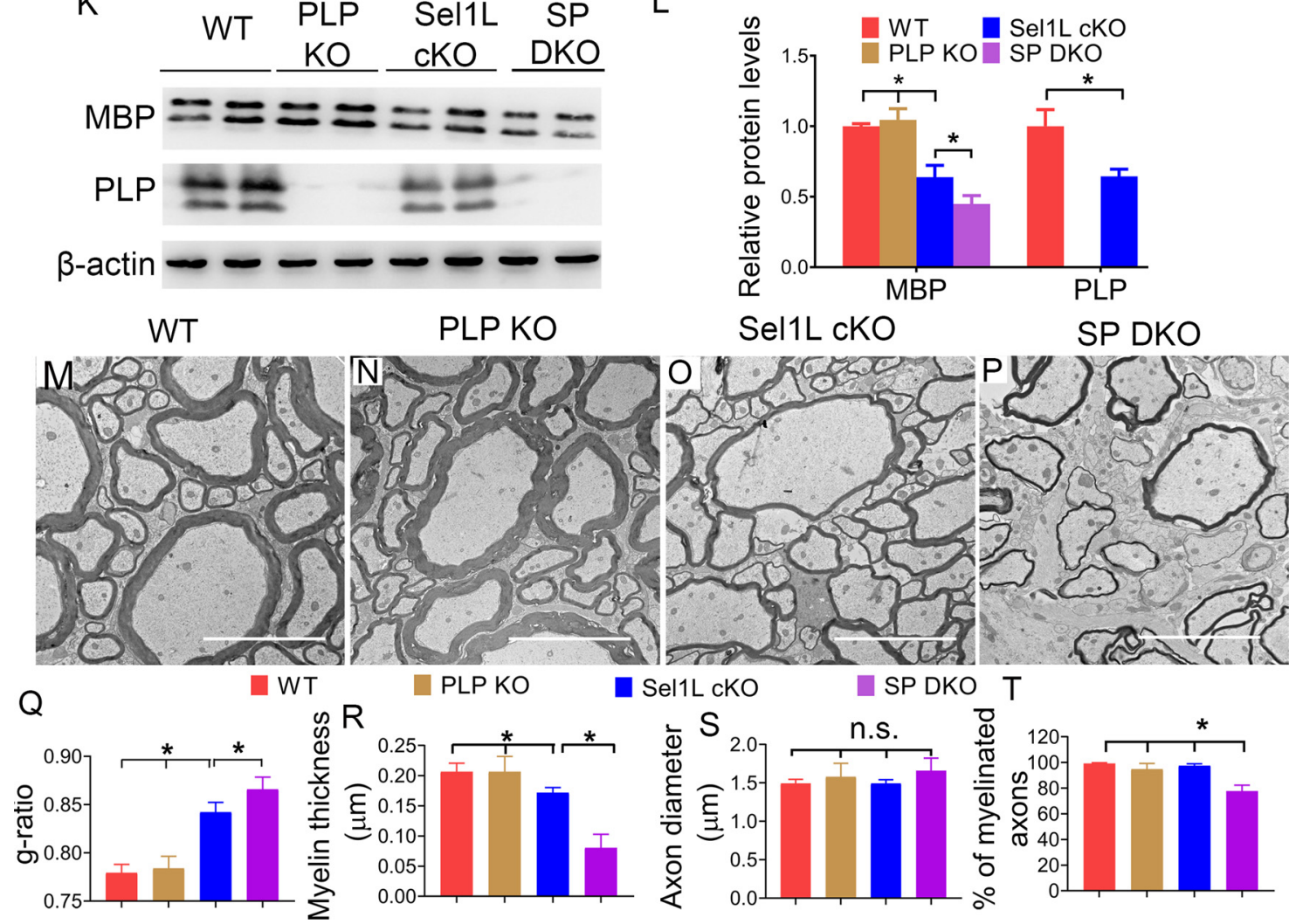

Figure 10. PLP deficiency exacerbated myelin thinning in the CNS of adult Sel1L cKO mice. A, Mouse survival curve showed that WT mice and PLP K0 mice survive at least 1 year. All Sel1L cK0 mice died by 43 weeks. Importantly, all SP DKO mice died by 16 weeks. $N=8$ animals. $\boldsymbol{B}$, Rotarod test showed that the latency to fall of Sel1L cKO mice was significantly shorter than WT mice starting at the age of 8 weeks, and that the latency to fall of SP DKO mice was significantly shorter than Sel1L cK0 starting at the age of 11 weeks (significant effect of group, $F_{(3,22)}=33.49$; significant effect of age, $F_{(6.018,132.4)}=7.886$; and significant group $\times$ age interaction, $\left.F_{(3242)}=3.398\right) . N=6-8$ animals. $(-J$, MBP IHC showed that the immunoreactivity of MBP is noticeably decreased in the spinal cord and brain of 10-week-old Sel1L cKO mice compared with PLP KO mice and WT mice. PLP deficiency further reduced the immunoreactivity of MBP in Sel1L cKO mice. $K, L$, Western blot analysis showed that PLP was undetectable in the brain of 10-week-old PLP K0 mice and SP DKO mice. The level of MBP was significantly decreased in the brain of 10-week-old Sel1L cK0 mice compared with PLP K0 mice and WT mice, and was further reduced in SP DKO mice. $M-T$, EM analysis showed a significant increase in the g-ratio $\left(F_{(3,12)}=58.66\right)$ and a significant reduction in myelin thickness $\left(F_{(3,12)}=39.77\right)$ in the spinal cord of 10-week-old Sel1L cKO mice compared with PLP KO mice and WT mice. PLP deficiency further increased the g-ratio and further reduced myelin thickness in Sel1L cK0 mice. Moreover, the percentage of myelinated axons was significantly decreased in the spinal cord of 10-week-old SP DKO mice compared with Sel1L cKO mice, PLP KO mice, and WT mice $\left(F_{(3,12)}=35.85\right)$. Conversely, the diameter of axons was comparable in the spinal cord of 10-week-old SP DKO mice, Sel1L cKO mice, PLP KO mice, and WT mice $\left(F_{(3,12)}=1.560\right)$. Scale bars: $M$-P, $5 \mu$ m. $N=4$ animals. Statistical analyses were done with a one-way ANOVA with a Tukey's post-test or a two-way ANOVA with a Bonferroni post-test. Error bars indicate SD. ${ }^{*} p<0.05$. n.s. not significant. 

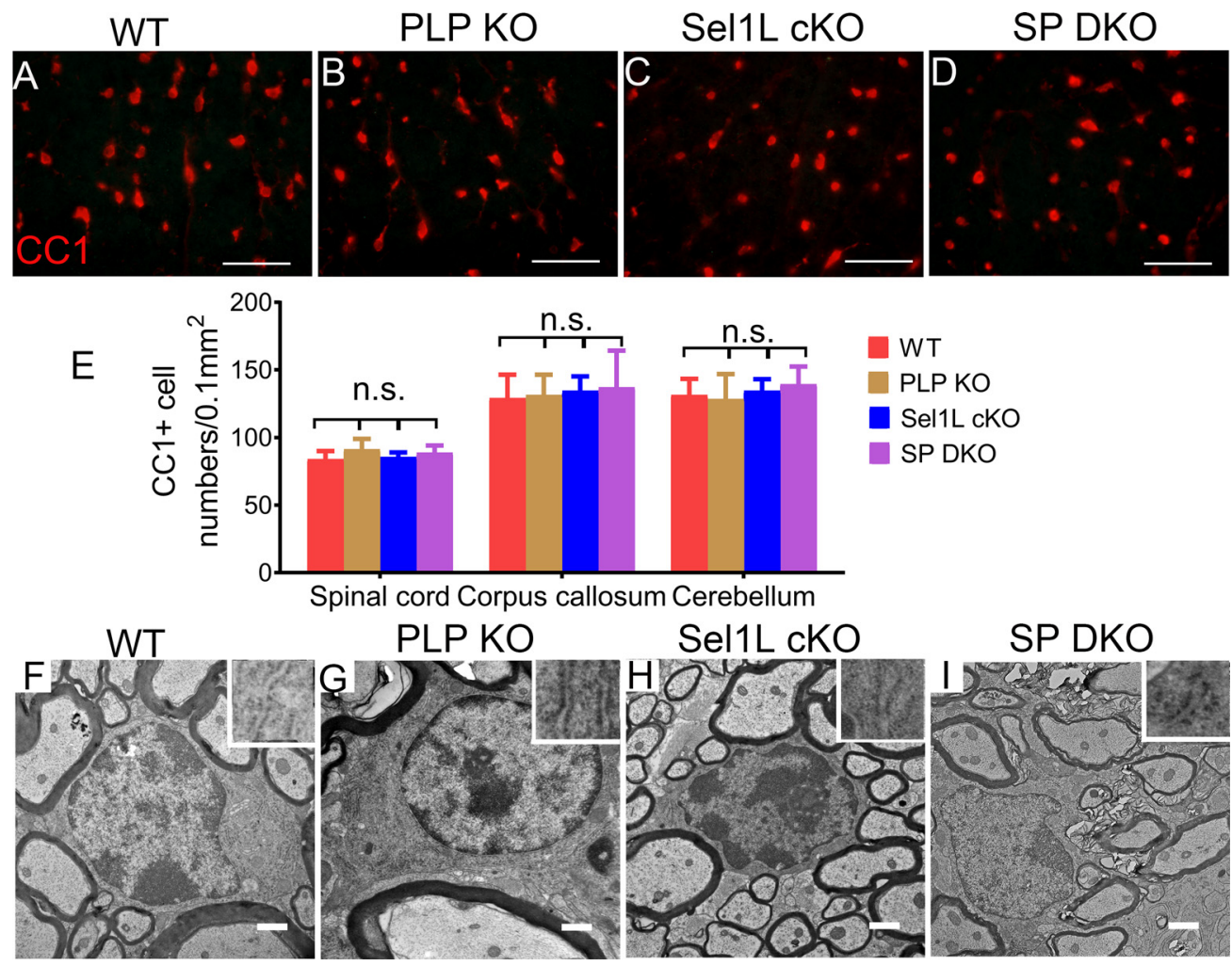

Figure 11. PLP deficiency did not affect oligodendrocyte viability in the CNS of adult Sel1L cKO mice. $A-E$, CC1 immunostaining showed a comparable number of oligodendrocytes in the spinal cord $\left(F_{(3,12)}=1.238\right)$, corpus callosum $\left(F_{(3,12)}=0.144\right)$, and cerebellum $\left(F_{(3,12)}=0.478\right)$ of 10-week-old SP DKO mice, Sel1L cKO mice, PLP KO mice, and WT mice. $\boldsymbol{F}-I$, EM analysis showed that the morphology of oligodendrocytes and the morphology of the ER (insets) in oligodendrocytes appeared normal in the spinal cord of 10-week-old SP DKO mice, Sel1L cKO mice, PLP K0 mice, and WT mice. Scale bars: $\mathbf{A}-\mathbf{D}, 50 \mu \mathrm{m} ; \boldsymbol{F}-\mathbf{I}, 1 \mu \mathrm{m}$. $N=4$ animals. Statistical analyses were done with a one-way ANOVA with a Tukey's post-test. Error bars indicate SD. n.s. not significant.

callosum, $p=0.9314$; cerebellum, $p=0.7031$ ). EM analysis also showed that both the morphology of oligodendrocytes and the morphology of the ER in these cells in 10-week-old SP DKO mice were indistinguishable from those of Sel1L cKO mice, PLP KO mice, and WT mice (Fig. $11 F-I$ ). These results demonstrate that PLP deficiency exacerbates myelin thinning induced by Sel1L deficiency in oligodendrocytes in adult mice. Collectively, these data provide additional evidence supporting that the reduction of myelin proteins is a major contributor to myelin thinning induced by Sel1L deficiency in oligodendrocytes in adult mice. These data also suggest that the Sel1L-Hrd1 complex is not a major player involved in PLP degradation, and rule out the possibility that intracellular accumulation of PLP contributes to mature oligodendrocyte dysfunction and death in adult Sel1L cKO mice.

\section{Discussion}

ERAD interacts with the UPR to maintain ER protein homeostasis (Nakatsukasa et al., 2014; Hetz et al., 2015; Qi et al., 2017; Hwang and Qi, 2018). The UPR controls the expression of ERAD genes. ERAD can regulate the UPR through direct degradation of ER stress sensors and/or indirect modulation of ER stress. Oligodendrocytes must produce an enormous amount of myelin proteins via the ER to assemble and maintain myelin structure (W. Lin and Popko, 2009; W. Lin and Stone, 2020). We showed here that Sel1L deficiency impaired the ERAD activity of the Sel1L-Hrd1 complex and induced moderate ER stress in oligodendrocytes, as evidenced by the UPR activation and the normal morphology of the ER in these cells. It was thought that actively myelinating oligodendrocytes during developmental myelination are highly sensitive to disruption of ER protein homeostasis because of the rate of myelin protein production (W. Lin and Popko, 2009; Volpi et al., 2017; W. Lin and Stone, 2020). Nevertheless, a very recent study showed that the impaired UPR in oligodendrocytes via deletion of both PERK and ATF6 $\alpha$ has a minimal effect on actively myelinating oligodendrocytes during developmental myelination but leads to dysfunction and death of mature oligodendrocytes in young adult mice (Stone et al., 2020). Accordingly, we found that Sel1L deficiency specifically in oligodendrocytes did not affect the viability or function of actively myelinating oligodendrocytes during developmental myelination. Moreover, we found that double deficiency of Sel1L and PERK did not alter the morphology of the ER in actively myelinating oligodendrocytes or their viability and function during developmental myelination. Together, these data indicate that the integrated UPR and ERAD are not a major player in maintaining ER protein homeostasis or the viability and function of actively myelinating oligodendrocytes during developmental myelination.

We found that Sel1L deficiency specifically in oligodendrocytes induced progressive thinning of myelin sheaths in the CNS of adult mice starting around the age of 6 weeks, and eventually led to complete loss of myelin sheath and oligodendrocyte death. We also found that the myelin thinning was coupled with the decreased levels of myelin proteins. We further ruled out the possibility that thin myelin in the CNS of adult Sel1L cKO mice was caused by oligodendrocyte regeneration and remyelination. Moreover, we showed activation of the PERK-eIF2 $\alpha$ pathway and attenuation of myelin protein biosynthesis in mature oligodendrocytes of adult Sel1L cKO mice. The PERK-eIF2 $\alpha$ pathway is the master regulator of protein translation in ER-stressed cells 
(Marciniak and Ron, 2006; Hetz et al., 2015; Wang and Kaufman, 2016). Interestingly, we found that PERK inactivation restored biosynthesis of myelin proteins in mature oligodendrocytes and the levels of myelin proteins in the CNS of adult Sel1L cKO mice. Importantly, PERK inactivation in oligodendrocytes attenuated the tremoring phenotype, prolonged the survival, and rescued progressive myelin thinning and oligodendrocyte death in the CNS of adult Sel1L cKO mice. Thus, these results suggest that PERK activation induced by SellL deficiency suppresses myelin protein translation in mature oligodendrocytes, leads to a reduction in myelin protein levels, and subsequently results in progressive myelin thinning in adult mice. Together, these data demonstrate that the integrated UPR and ERAD are required for maintaining myelin protein production and the viability and function of mature oligodendrocytes in adult mice.

Myelin proteins are essential and necessary for maintaining myelin structures (Baumann and Pham-Dinh, 2001; Aggarwal et al., 2011; Stadelmann et al., 2019). Our data support the notion that myelin thinning in the CNS of adult Sel1L cKO mice results from the reduction of myelin proteins. To further explore this notion, we exploited PLP KO mice that display very subtle myelin abnormality in young adult mice and survive well (Klugmann et al., 1997; Griffiths et al., 1998; Rosenbluth et al., 2006). Interestingly, we found that PLP deficiency aggravated the tremoring phenotype, shortened the lifespan, and exacerbated myelin thinning in adult Sel1L cKO mice. Conversely, PLP deficiency did not alter the morphology or number of oligodendrocytes in adult Sel1L cKO mice. Collectively, these data provide additional evidence supporting that the reduction of myelin proteins is a major contributor to myelin thinning in the CNS of adult Sel1L cKO mice. Nevertheless, mice with a deficiency of an individual myelin protein, including PLP, $2^{\prime}, 3^{\prime}$ cyclic nucleotide $3^{\prime}$ phosphodiesterase, myelin oligodendrocyte glycoprotein, or myelinassociated glycoprotein, do not display obvious myelin abnormalities at young age (Montag et al., 1994; Klugmann et al., 1997; Delarasse et al., 2003; Lappe-Siefke, et al., 2003), although MBP-deficient mice develop severe myelin loss in the CNS (Popko et al., 1987). We showed here that there was a global reduction in myelin proteins, including at least MBP and PLP, in the CNS of adult Sel1L cKO mice. Thus, our data likely reflect that the global reduction of myelin proteins, rather than a reduction in individual myelin proteins, contributes to myelin thinning in the CNS of adult Sel1L cKO mice. On the other hand, it is likely that PERK activation suppresses global protein translation in mature oligodendrocytes of adult Sel1L cKO mice, including myelin proteins, prosurvival proteins, among others. The possibility that impaired translation of nonmyelin proteins in mature oligodendrocytes contributes to myelin thinning in the CNS of adult Sel1L cKO mice cannot be ruled out.

The finding of the detrimental effects of PERK activation on mature oligodendrocytes in adult Sel1L cKO mice contradicts previous studies that show the beneficial effects of PERK activation on oligodendrocytes in immune-mediated demyelinating diseases (W. Lin et al., 2005, 2006, 2007, 2013; Y. Lin et al., 2014b; Hussien et al., 2014). Conversely, a previous study showed that short-term, artificially strong PERK activation in mature oligodendrocytes does not affect their viability but causes moderate myelin loss in adult mice (Y. Lin et al., 2014a). Interestingly, data indicate that the beneficial or detrimental effects of PERK activation on cell viability and function are highly context-dependent in the degree and/or duration of the activation (Y. Lin et al., 2014a; Stone et al., 2016; McQuiston and Diehl et al., 2017; W. Lin and Stone, 2020). It is possible that the short-term (days) PERK activation promotes oligodendrocyte survival in acute immune-mediated demyelinating diseases by stimulating cytoprotective gene expression; however, long-term (weeks or months) PERK activation leads to depletion of myelin proteins and/or prosurvival proteins in oligodendrocytes by suppressing global protein translation, and subsequently results in dysfunction and death of mature oligodendrocytes in adult Sel1L cKO mice. Similarly, short-term, artificially strong PERK activation in oligodendrocytes dramatically reduces protein translation, leads to depletion of myelin proteins, and results in myelin loss but not oligodendrocyte death in the CNS of adult mice. The alternative, but not mutually exclusive, possibility is that the detrimental effects of PERK activation on mature oligodendrocytes in adult Sel1L cKO mice are because of the unique cellular context of Sel1L-deficient oligodendrocytes.

The current dogma is that myelin thickness in the adult CNS is extraordinarily stable (Aggarwal et al., 2011; Stadelmann et al., 2019). Indeed, using thin myelin as a marker of newly generated myelin (remyelination) in CNS demyelinating diseases is a wellaccepted practice (Franklin and Ffrench-Constant, 2008, 2017; Duncan et al., 2018). Nevertheless, very recent studies show that myelin thickness can change dynamically in adults over time (Dutta et al., 2018; Williamson and Lyons, 2018; Fields and Dutta, 2019; Phillips et al., 2019). Accordingly, the results presented in this study suggest that PERK activation in mature oligodendrocytes reduces myelin thickness by suppressing myelin protein translation in adult Sel1 $\mathrm{L} \mathrm{cKO}$ mice. Interestingly, it is well documented that PERK is activated in oligodendrocytes in various CNS demyelinating diseases (W. Lin and Popko, 2009; Volpi et al., 2017; W. Lin and Stone, 2020). Thus, this study raises the possibility that PERK activation in mature oligodendrocytes can lead to myelin thinning in CNS demyelinating diseases, and that thin myelin may not be a reliable marker of remyelination in these diseases. Future studies to determine whether PERK activation in mature oligodendrocytes causes myelin thinning in CNS demyelinating diseases are warranted.

In conclusion, we showed here that Sel1L deficiency specifically in oligodendrocytes did not affect developmental myelination, but led to late-onset, progressive myelin thinning in adult mice. The progressive myelin thinning induced by Sel1L deficiency in oligodendrocytes was associated with PERK activation and attenuation of myelin protein translation, and was abrogated by PERK inactivation in oligodendrocytes. Moreover, the progressive myelin thinning induced by Sel1L deficiency in oligodendrocytes was coupled with the reduction of myelin proteins, and was exacerbated by PLP deficiency. These findings imply that the integrated UPR and ERAD in mature oligodendrocytes are required for preserving myelin thickness in adults by regulating myelin protein biosynthesis.

\section{References}

Aggarwal S, Yurlova L, Simons M (2011) Central nervous system myelin: structure, synthesis and assembly. Trends Cell Biol 21:585-593.

Baumann N, Pham-Dinh D (2001) Biology of oligodendrocyte and myelin in the mammalian central nervous system. Physiol Rev 81:871-927.

Chow E, Mottahedeh J, Prins M, Ridder W, Nusinowitz S, Bronstein JM (2005) Disrupted compaction of CNS myelin in an OSP/Claudin-11 and PLP/DM20 double knockout mouse. Mol Cell Neurosci 29:405-413.

Delarasse C, Daubas P, Mars LT, Vizler C, Litzenburger T, Iglesias A, Bauer J, Della Gaspera B, Schubart A, Decker L, Dimitri D, Roussel G, Dierich A, Amor S, Dautigny A, Liblau R, Pham-Dinh D (2003) Myelin/oligodendrocyte glycoprotein-deficient (MOG-deficient) mice reveal lack of immune tolerance to MOG in wild-type mice. J Clin Invest 112:544-553 
Duncan ID, Radcliff AB, Heidari M, Kidd G, August BK, Wierenga LA (2018) The adult oligodendrocyte can participate in remyelination. Proc Natl Acad Sci USA 115:E11807-E11816.

Dutta DJ, Woo DH, Lee PR, Pajevic S, Bukalo O, Huffman WC, Wake H, Basser PJ, SheikhBahaei S, Lazarevic V, Smith JC, Fields RD (2018) Regulation of myelin structure and conduction velocity by perinodal astrocytes. Proc Natl Acad Sci USA 115:11832-11837.

Fields RD, Dutta DJ (2019) Treadmilling model for plasticity of the myelin sheath. Trends Neurosci 42:443-447.

Franklin RJ, Ffrench-Constant C (2008) Remyelination in the CNS: from biology to therapy. Nat Rev Neurosci 9:839-855.

Franklin RJ, Ffrench-Constant C (2017) Regenerating CNS myelin: from mechanisms to experimental medicines. Nat Rev Neurosci 18:753-769.

Griffiths IR, Schneider A, Anderson J, Nave KA (1995) Transgenic and natural mouse models of proteolipid protein (PLP)-related dysmyelination and demyelination. Brain Pathol 5:275-281.

Griffiths I, Klugmann M, Anderson T, Yool D, Thomson C, Schwab MH, Schneider A, Zimmermann F, McCulloch M, Nadon N, Nave KA (1998) Axonal swellings and degeneration in mice lacking the major proteolipid of myelin. Science 280:1610-1613.

Goodman CA, Mabrey DM, Frey JW, Miu MH, Schmidt EK, Pierre P, Hornberger TA (2011) Novel insights into the regulation of skeletal muscle protein synthesis as revealed by a new nonradioactive in vivo technique. FASEB J 25:1028-1039.

Hetz C, Chevet E, Oakes SA (2015) Proteostasis control by the unfolded protein response. Nat Cell Biol 17:829-838.

Horimoto S, Ninagawa S, Okada T, Koba H, Sugimoto T, Kamiya Y, Kato K, Takeda S, Mori K (2013) The unfolded protein response transducer ATF6 represents a novel transmembrane-type endoplasmic reticulumassociated degradation substrate requiring both mannose trimming and SEL1L protein. J Biol Chem 288:31517-31527.

Hussien Y, Cavener DR, Popko B (2014) Genetic inactivation of PERK signaling in mouse oligodendrocytes: normal developmental myelination with increased susceptibility to inflammatory demyelination. Glia 62:680-691.

Hussien Y, Podojil JR, Robinson AP, Lee AS, Miller SD, Popko B (2015) ER chaperone $\mathrm{BiP} / \mathrm{GRP} 78$ is required for myelinating cell survival and provides protection during experimental autoimmune encephalomyelitis. J Neurosci 35:15921-15933.

Hwang J, Qi L (2018) Quality control in the endoplasmic reticulum: crosstalk between ERAD and UPR pathways. Trends Biochem Sci 43:593-605.

Kang SH, Fukaya M, Yang JK, Rothstein JD, Bergles DE (2010) $\mathrm{NG}^{2+}$ CNS glial progenitors remain committed to the oligodendrocyte lineage in postnatal life and following neurodegeneration. Neuron 68:668-681.

Karim SA, Barrie JA, McCulloch MC, Montague P, Edgar JM, Iden DL, Anderson TJ, Nave KA, Griffiths IR, McLaughlin M (2010) PLP/DM20 expression and turnover in a transgenic mouse model of PelizaeusMerzbacher disease. Glia 58:1727-1738.

Klugmann M, Schwab MH, Pühlhofer A, Schneider A, Zimmermann F, Griffiths IR, Nave KA (1997) Assembly of CNS myelin in the absence of proteolipid protein. Neuron 18:59-70.

Lappe-Siefke C, Goebbels S, Gravel M, Nicksch E, Lee J, Braun PE, Griffiths IR, Nave KA (2003) Disruption of Cnp1 uncouples oligodendroglial functions in axonal support and myelination. Nat Genet 33:366-374.

Lee CS, Hanna AD, Wang H, Dagnino-Acosta A, Joshi AD, Knoblauch M, Xia Y, Georgiou DK, Xu J, Long C, Amano H, Reynolds C, Dong K, Martin JC, Lagor WR, Rodney GG, Sahin E, Sewry C, Hamilton SL (2017) A chemical chaperone improves muscle function in mice with a RyR1 mutation. Nat Commun 8:14659.

Lin W (2015) Impaired eIF2B activity in oligodendrocytes contributes to VWMD pathogenesis. Neural Regen Res 10:195-197.

Lin W, Popko B (2009) Endoplasmic reticulum stress in disorders of myelinating cells. Nat Neurosci 12:379-385.

Lin W, Stone S (2020) Unfolded protein response in myelin disorders. Neural Regen Res 15:636-645.

Lin W, Harding HP, Ron D, Popko B (2005) Endoplasmic reticulum stress modulates the response of myelinating oligodendrocytes to the immune cytokine interferon-gamma. J Cell Biol 169:603-612.

Lin W, Kemper A, Dupree JL, Harding HP, Ron D, Popko B (2006) Interferon-gamma inhibits central nervous system remyelination through a process modulated by endoplasmic reticulum stress. Brain 129:13061318.
Lin W, Bailey SL, Ho H, Harding HP, Ron D, Miller SD, Popko B (2007) The integrated stress response prevents demyelination by protecting oligodendrocytes against immune-mediated damage. J Clin Invest 117:448456.

Lin W, Lin Y, Li J, Fenstermaker AG, Way SW, Clayton B, Jamison S, Harding HP, Ron D, Popko B (2013) Oligodendrocyte-specific activation of PERK signaling protects mice against experimental autoimmune encephalomyelitis. J Neurosci 33:5980-5991.

Lin Y, Pang X, Huang G, Jamison S, Fang J, Harding HP, Ron D, Lin W (2014a) Impaired eukaryotic translation initiation factor 2B activity specifically in oligodendrocytes reproduces the pathology of vanishing white matter disease in mice. J Neurosci 34:12182-12191.

Lin Y, Huang G, Jamison S, Li J, Harding HP, Ron D, Lin W (2014b) PERK activation preserves the viability and function of remyelinating oligodendrocytes in immune-mediated demyelinating diseases. Am J Pathol 184:507-519.

Marciniak SJ, Ron D (2006) Endoplasmic reticulum stress signaling in disease. Physiol Rev 86:1133-1149.

McQuiston A, Diehl JA (2017) Recent insights into PERK-dependent signaling from the stressed endoplasmic reticulum. F1000Res 6:1897.

Montag D, Giese KP, Bartsch U, Martini R, Lang Y, Blüthmann $\mathrm{H}$, Karthigasan J, Kirschner DA, Wintergerst ES, Nave KA, Zielasek J, Toyka KV, Lipp H, Schachner M (1994) Mice deficient for the myelin-associated glycoprotein show subtle abnormalities in myelin. Neuron 13:229-246.

Nakatsukasa K, Kamura T, Brodsky JL (2014) Recent technical developments in the study of ER-associated degradation. Curr Opin Cell Biol 29:82-91.

Pavitt GD, Proud CG (2009) Protein synthesis and its control in neuronal cells with a focus on vanishing white matter disease. Biochem Soc Trans 37:1298-12310.

Pavitt GD, Ron D (2012) New insights into translational regulation in the endoplasmic reticulum unfolded protein response. Cold Spring Harb Perspect Biol 4:a012278.

Phillips KA, Watson CM, Bearman A, Knippenberg AR, Adams J, Ross C, Tardif SD (2019) Age-related changes in myelin of axons of the corpus callosum and cognitive decline in common marmosets. Am J Primatol 81:e22949.

Popko B, Puckett C, Lai E, Shine HD, Readhead C, Takahashi N, Hunt SW, Sidman RL, Hood L (1987) Myelin deficient mice: expression of myelin basic protein and generation of mice with varying levels of myelin. Cell 48:713-721.

Psachoulia K, Jamen F, Young KM, Richardson WD (2009) Cell cycle dynamics of NG2 cells in the postnatal and ageing brain. Neuron Glia Biol 5:57-67.

Qi L, Tsai B, Arvan P (2017) New insights into the physiological role of endoplasmic reticulum-associated degradation. Trends Cell Biol 27:430-440.

Rivers LE, Young KM, Rizzi M, Jamen F, Psachoulia K, Wade A, Kessaris N, Richardson WD (2008) PDGFRA/NG2 glia generate myelinating oligodendrocytes and piriform projection neurons in adult mice. Nat Neurosci 11:1392-1401.

Rosenbluth J, Nave KA, Mierzwa A, Schiff R (2006) Subtle myelin defects in PLP-null mice. Glia 54:172-182.

Ruggiano A, Foresti O, Carvalho P (2014) Quality control: ER-associated degradation: protein quality control and beyond. J Cell Biol 204:869-879.

Sabri MI, Bone AH, Davison AN (1974) Turnover of myelin and other structural proteins in the developing rat brain. Biochem J 142:499-507.

Schmidt EK, Clavarino G, Ceppi M, Pierre P (2009) SUnSET, a nonradioactive method to monitor protein synthesis. Nat Methods 6:275-277.

Stadelmann C, Timmler S, Barrantes-Freer A, Simons M (2019) Myelin in the central nervous system: structure. Physiol Rev 99:1381-1431.

Stone S, Ho Y, Li X, Jamison S, Harding HP, Ron D, Lin W (2016) Dual role of the integrated stress response in medulloblastoma tumorigenesis. Oncotarget 7:64124-64135.

Stone S, Jamison S, Yue Y, Durose W, Schmidt-Ullrich R, Lin W (2017) NF$\kappa \mathrm{B}$ activation protects oligodendrocytes against inflammation. J Neurosci 37:9332-9344.

Stone S, Wu S, Jamison S, Durose W, Pallais JP, Lin W (2018) Activating transcription factor $6 \alpha$ deficiency exacerbates oligodendrocyte death and myelin damage in immune-mediated demyelinating diseases. Glia 66:1331-1345

Stone S, Wu S, Nave KA, Lin W (2020) The UPR preserves mature oligodendrocyte viability and function in adults by regulating autophagy of PLP. JCI Insight 5 5:e132364. 
Sun S, Shi G, Han X, Francisco AB, Ji Y, Mendonça N, Liu X, Locasale JW, Simpson KW, Duhamel GE, Kersten S, Yates JR, Long Q, Qi L (2014) Sel1L is indispensable for mammalian endoplasmic reticulum-associated degradation, endoplasmic reticulum homeostasis, and survival. Proc Natl Acad Sci USA 111:E582-E591.

Sun S, Shi G, Sha H, Ji Y, Han X, Shu X, Ma H, Inoue T, Gao B, Kim H, Bu P, Guber RD, Shen X, Lee AH, Iwawaki T, Paton AW, Paton JC, Fang D, Tsai B, Yates JR, et al. (2015) IRE $1 \alpha$ is an endogenous substrate of endoplasmic-reticulum-associated degradation. Nat Cell Biol 17:1546-1555.

Sugimoto T, Ninagawa S, Yamano S, Ishikawa T, Okada T, Takeda S, Mori K (2017) SEL1L-dependent substrates require Derlin2/3 and Herp1/2 for endoplasmic reticulum-associated degradation. Cell Struct Funct 42:81-94.

Uschkureit T, Sporkel O, Stracke J, Bussow H, Stoffel W (2000) Early onset of axonal degeneration in double (plp-/-mag-/-) and hypomyelinosis in triple (plp-/-mbp-/-mag-/-) mutant mice: early onset of axonal degeneration in double (plp-/-mag-/-) and hypomyelinosis in triple (plp-/-mbp-/mag-/-) mutant mice. J Neurosci 20:5225-5233.

Volpi VG, Touvier T, D’Antonio M (2017) Endoplasmic reticulum protein quality control failure in myelin disorders. Front Mol Neurosci 9:162.
Wang M, Kaufman RJ (2016) Protein misfolding in the endoplasmic reticulum as a conduit to human disease. Nature 529:326-335.

Williamson JM, Lyons DA (2018) Myelin dynamics throughout life: an everchanging landscape? Front Cell Neurosci 12:424.

Woodward KJ (2008) The molecular and cellular defects underlying Pelizaeus-Merzbacher disease. Expert Rev Mol Med 10:e14

Wu X, Rapoport TA (2018) Mechanistic insights into ER-associated protein degradation. Curr Opin Cell Biol 53:22-28.

Yoon SB, Park YH, Choi SA, Yang HJ, Jeong PS, Cha JJ, Lee S, Lee SH, Lee JH, Sim BW, Koo BS, Park SJ, Lee Y, Kim YH, Hong JJ, Kim JS, Jin YB, Huh JW, Lee SR, Song BS, et al. (2019) Real-time PCR quantification of spliced X-box binding protein 1 (XBP1) using a universal primer method. PLoS One 14:e0219978.

Zhang P, McGrath B, Li S, Frank A, Zambito F, Reinert J, Gannon M, Ma K, McNaughton K, Cavener DR (2002) The PERK eukaryotic initiation factor 2 alpha kinase is required for the development of the skeletal system, postnatal growth, and the function and viability of the pancreas. Mol Cell Biol 22:3864-3874. 